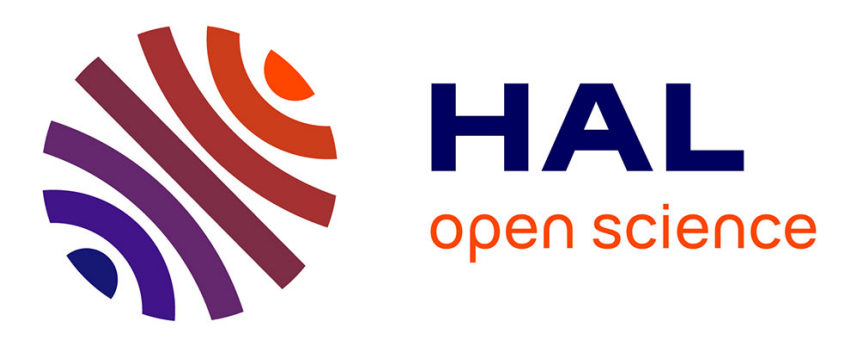

\title{
Singularity Analysis of 3T2R Parallel Mechanisms using Grassmann-Cayley Algebra and Grassmann Line Geometry
}

Semaan Amine, M Tale Masouleh, Stéphane Caro, Philippe Wenger, Clément Gosselin

\section{- To cite this version:}

Semaan Amine, M Tale Masouleh, Stéphane Caro, Philippe Wenger, Clément Gosselin. Singularity Analysis of 3T2R Parallel Mechanisms using Grassmann-Cayley Algebra and Grassmann Line Geometry. Mechanism and Machine Theory, 2012, 52, pp.326-340. 10.1016/j.mechmachtheory.2011.11.015 . hal-00833520

\section{HAL Id: hal-00833520 \\ https://hal.science/hal-00833520}

Submitted on 12 Jun 2013

HAL is a multi-disciplinary open access archive for the deposit and dissemination of scientific research documents, whether they are published or not. The documents may come from teaching and research institutions in France or abroad, or from public or private research centers.
L'archive ouverte pluridisciplinaire HAL, est destinée au dépôt et à la diffusion de documents scientifiques de niveau recherche, publiés ou non, émanant des établissements d'enseignement et de recherche français ou étrangers, des laboratoires publics ou privés. 


\title{
Singularity Analysis of 3T2R Parallel Mechanisms using Grassmann-Cayley Algebra and Grassmann Geometry
}

\author{
S. Amine* \\ M. Tale Masouleh ${ }^{\dagger}$ \\ S. Caro \\ P. Wenger ${ }^{\S}$ \\ C. Gosselin $\llbracket$ \\ IRCCyN \\ Université Laval \\ IRCCyN \\ IRCCyN \\ Université Laval \\ Nantes, France \\ Québec, Canada \\ Nantes, France \\ Nantes, France \\ Québec, Canada
}

June 12, 2013

\begin{abstract}
This paper deals with the singular configurations of symmetric 5-DOF parallel mechanisms performing three translational and two independent rotational DOFs. The screw theory approach is adopted in order to obtain the Jacobian matrices. The regularity of these matrices is examined using Grassmann-Cayley algebra and Grassmann geometry. More emphasis is placed on the geometric investigation of singular configurations by means of Grassmann-Cayley Algebra for a class of simplified designs whereas Grassmann geometry is used for a matter of comparison. The results provide algebraic expressions for the singularity conditions, in terms of some bracket monomials obtained from the superbracket decomposition. Accordingly, all the singularity conditions can be enumerated.
\end{abstract}

Keywords: Parallel singularity, screw theory, 5-DOF parallel mechanisms, Grassmann geometry, GrassmannCayley algebra, Jacobian matrix, superbracket decomposition

\section{Introduction}

The singular configurations of Parallel Mechanisms (PMs) are critical poses characterized by either the loss of some degrees of freedom (DOF), the gain of some extra DOF or the loss of stiffness. The determination of singular configurations is thus a central issue in robotics due to their major effect on the robot performance [1, 2]. Lower-mobility PMs are suitable for a wide range of applications that require fewer than six DOF. The classification of the singularities of lower-mobility PMs has stimulated the interest of many researchers [3-6]. In this paper, the classification proposed in [4] is adopted. Accordingly, a lower-mobility PM can exhibit three types of singularities: ( $i$ ) limb singularities, $(i i)$ platform singularities [4], also known as constraint singularities [7] and (iii) actuation singularities. In this paper, more emphasis is placed on the actuation singularities that are more challenging than the other ones for the mechanisms under study.

Constraint and actuation singularities are referred to as parallel singularities and are related to the rank deficiency of a Jacobian matrix derived from the first-order kinematic relation. The latter relation is referred to as the input-output velocity equation and is linear with respect to the time-rate changes of the input-output variables. The determination of the parallel singularities of a PM consists in finding either the poses, yielding the singularity locus, or the conditions, yielding the configurations, in which the Jacobian matrix becomes rankdeficient. A $6 \times 6$ Jacobian matrix $\mathbf{J}$ of a given lower-mobility PM can be derived following the methodology proposed in [8] based on screw theory.

The next step is to explore the regularity of $\mathbf{J}$, which can be performed with two general approaches: linear algebra and Grassmann geometry [1, 2, 9,-16]. Linear algebra consists in a direct analysis of $\mathbf{J}$ by expanding its determinant and examining its vanishing conditions. Generally, the determinant of such a matrix is highly

\footnotetext{
*semaan.amine@irccyn.ec-nantes.fr

† mehdi.tale-masouleh.1@ulaval.ca

¥stephane.caro@irccyn.ec-nantes.fr

$\S$ philippe.wenger@irccyn.ec-nantes.fr

Tgosselin@gmc.ulaval.ca
} 
non linear and unwieldy to assess, even with a computer algebra system. Hence, linear algebra fails to provide satisfactory results, and therefore, the use of Grassmann-Cayley Algebra (GCA) [2, 11, 12, 14] or Grassmann Geometry (GG) [1, 9, 10, 13, 16] may be regarded as promising solutions to explore the degeneracy of $\mathbf{J}$, namely, to analyze the singularities of PMs.

The GCA is a systematic approach to obtain a bracket representation of the determinant of $\mathbf{J}$, called $s u$ perbracket. By exploring this superbracket, it is possible to obtain a geometrical interpretation of the parallel singularities. On the other hand, GG is a geometric approach that provides a classification for the conditions under which a set of $n$ Plücker lines spans a variety of dimension lower than $n$. This paper focuses on the application of GCA to provide a compact vector expression for the singularity locus of 3T1R PMs with identical limb structures.

Symmetrid 1 5-DOF PMs performing three translational and two independent rotational motions, referred to as $3 \mathrm{~T} 2 \mathrm{R}$, are considered as case studies to apply the GCA for two main reasons:

1. Recent studies on their kinematic properties, such as the Forward Kinematic Problem (FKP) [17-19], led to interesting results and this might also be the case for their singularity analysis;

2. The actuation wrench and the constraint wrench - a line at infinity-of a given limb of such mechanisms are not directly associated with the direction of a given mechanical joint (e.g., for the Gough-Stewart platform with 6-UPS as kinematic arrangement the actuation wrench is along the prismatic actuator direction).

In general, 5-DOF PMs fall into three classes according to their mobility: $(i)$ three translational and two rotational DOFs (3T2R), (ii) three rotational and two planar translational DOFs $\left(3 \mathrm{R} 2 \mathrm{~T}_{\mathrm{p}}\right)$ where the planar motion could be either fixed or instantaneous and (iii) three rotational and two spherical translational DOFs $\left(3 \mathrm{R} 2 \mathrm{~T}_{\mathrm{s}}\right)$ [20]. Geometrically, the 3T2R motion can be made equivalent to guiding a combination of a directed line and a point on it. Accordingly, the 3T2R PMs can be used in a wide range of applications for a point-line combination including, among others, 5-axis machine tools [21], welding and conical spray-gun. Moreover, 5DOF PMs are very useful for medical applications [22] that require at the same time mobility, compactness and accuracy around a functional point. So far researchers have mainly worked on the type synthesis of such PMs [4, 20, 23-26]. There were no symmetrical 5-DOF PMs until Huang and Li and Jin et al. independently solved the problem and filled this gap [27, 28]. It is noteworthy that the most existing 5-DOF PMs are asymmetrical, i.e., have a 5-DOF passive limb that constrains some actuated 6-DOF limbs [29,30].

Due to the short history of symmetric 5-DOF PMs, more precisely the ones performing a 3T2R motion pattern, their kinematic properties are still not well understood. In some recent studies, the Inverse Kinematic Problem (IKP), Forward Kinematic Problem (FKP), workspace and singularities were investigated [18, 19, 3134]. The singularities were investigated upon a different perspective based on GG [32]. The results obtained in [32] are based on some inspections and intuitions. Thus, as a global objective, this paper aims at blending these two approaches, namely, GCA and GG in order to obtain a more systematic approach to enumerate all the singular configurations of the PMs under study. These PMs originate from the comprehensive study of symmetric 5-DOF PMs (3T2R) whose FKP admits either a closed-form solution or a univariate expression [19, [34]. It should be noted that the GCA is applied based on the framework presented in [2, 11, 35,-37].

The remainder of the paper is organized as follows. First, some concepts for the singularity analysis are recalled. Then, the general architecture of the PMs under study is presented and a brief review of some existing kinematic properties is given, which leads us to a class of simplified designs with certain practical interests. In this paper, the singularity analysis method based on GCA is applied to two simplified designs. The results obtained with GCA are then put in contrast with the ones obtained recently using GG in order to make these two approaches complementary rather than opposite. Finally, the paper concludes with some remarks that provide insight into ongoing works to extend this study to other types of lower-mobility PMs.

\footnotetext{
${ }^{1}$ In the context of this paper, the term symmetric refers to the limb type and not to the geometry, such as centro-symmetrical simplifications.
} 


\section{Preliminary Concepts}

\subsection{Screw Theory}

Screw theory [37-40] is suitable for the type synthesis [20] and the study of the instantaneous motion [41] of PMs. A twist and a wrench are screws that represent the instantaneous motion of a rigid body and a system of forces and moments applied on a rigid body, respectively. A zero-pitch wrench, namely, a pure force, corresponds to the Plücker coordinate vector of a finite line in the 3-dimensional projective space $\mathbb{P}_{3}$. In turn, an infinite-pitch wrench, namely, a pure moment, corresponds to the Plücker coordinate vector of a line at infinity in $\mathbb{P}_{3}$. It is noteworthy that finite lines and lines at infinity are projective lines, i.e., Plücker lines whose six components satisfy the Grassmann Plücker relation [42]. A $n$-screw system is a screw subspace whose basis is composed of $n$ screws.

\subsection{Projective Space}

The 3-dimensional projective space $\mathbb{P}_{3}$ is characterized by the affine space $\mathbb{R}^{3}$ in addition to the plane at infinity $\Omega_{\infty}$. It is noteworthy that the coordinates of a projective element are determined up to a scalar multiple. A projective point has four homogeneous coordinates whereas a projective line has six Plücker coordinates represented by its Plücker coordinate vector. The following properties highlight the relations between projective elements:

1. A finite point, $A$, is represented by its homogeneous coordinates vector a $=\left(a_{1}, a_{2}, a_{3}, 1\right)^{T}$, the first three coordinates being its Cartesian coordinates in $\mathbb{R}^{3}$;

2. A finite line, $\mathcal{L}$, is represented by its Plücker coordinates vector $F=(\mathbf{s} ; \mathbf{r} \times \mathbf{s})$; where $\mathbf{s}$ is the line direction, $(\mathbf{r} \times \mathbf{s})$ represents the moment of $\mathcal{L}$ with respect to the origin and $\mathbf{r}$ is the position vector of any point on $\mathcal{L}$;

3. Let underlined points denote points at infinity. Any finite line, $F=(\mathbf{s} ; \mathbf{r} \times \mathbf{s})$, has a unique point at infinity $\underline{c}=(\mathbf{s} ; 0)$. This point is determined by the line direction up to a scalar multiple. Thus, all finite lines of direction $\mathbf{s}$ intersect at one common point at infinity, namely, $\underline{\mathbf{c}}$;

4. All finite planes of normal vector $\mathbf{m}$, have a common line at infinity. This line is given by: $M=(\mathbf{0} ; \mathbf{m})$ and passes through the point at infinity of any finite line orthogonal to $\mathbf{m}$;

5. Two lines at infinity $M_{1}=\left(\mathbf{0} ; \mathbf{m}_{1}\right)$ and $M_{2}=\left(\mathbf{0} ; \mathbf{m}_{2}\right)$ intersect at a unique point at infinity $\underline{\mathrm{g}}=$ $\left(\mathbf{m}_{1} \times \mathbf{m}_{2} ; 0\right)$.

\subsection{Grassmann-Cayley Algebra and Superbracket}

The GCA was developed by H. Grassmann (1809-1877) as a calculus for linear varieties operating on extensors with the join " $\vee$ " and meet " $\wedge$ " operators. The latter are associated with the span and intersection of vector spaces of extensors characterized with their step. GCA makes it possible to work at the symbolic level, and therefore, to produce coordinate-free algebraic expressions for the singularity conditions of spatial PMs. For further details on GCA, the reader is referred to [2,11,42] and references therein. The rows of $\mathbf{J}$ of a PM are usually Plücker coordinate vectors of six projective lines [8,43]. The superjoin of these six vectors in $\mathbb{P}_{5}$ corresponds to the determinant of $\mathbf{J}$ up to a scalar multiple, which is the superbracket in GCA $\Lambda\left(V^{(2)}\right)$ [42]. Thus, a singularity occurs when this superbracket vanishes. The superbracket is an expression involving 12 points selected on the six lines and can be developed into a linear combination of 24 bracket monomials [2, 44], each one being the product of three brackets of four projective points:

$$
[\mathrm{ab}, \mathrm{cd}, \mathrm{ef}, \mathrm{gh}, \mathrm{ij}, \mathrm{kl}]=\sum_{i=1}^{24} y_{i}
$$


where

$$
\begin{aligned}
& y_{1}=-[\mathrm{abcd}][\mathrm{efgi}][\mathrm{hjkl}] \quad y_{2}=\text { [abcd][efhi][gjkl] } \quad y_{3}=[\mathrm{abcd}][\mathrm{efgj}][\mathrm{hikl}] \\
& y_{4}=-[\mathrm{abcd}][\mathrm{efhj}][\mathrm{gikl}] \quad y_{5}=\text { [abce][dfgh][ijkl] } \quad y_{6}=- \text { [abde][cfgh][ijkl] } \\
& y_{7}=-[\mathrm{abcf}][\mathrm{degh}][\mathrm{ijkl}] \quad y_{8}=[\mathrm{abdf}][\mathrm{cegh}][\mathrm{ijkl}] \quad y_{9}=-[\mathrm{abce}][\mathrm{dghi}][\mathrm{fjkl}] \\
& y_{10}=[\mathrm{abde}][\mathrm{cghi}][\mathrm{fjkl}] \quad y_{11}=[\mathrm{abcf}][\mathrm{dghi}][\mathrm{ejkl}] \quad y_{12}=[\mathrm{abce}][\mathrm{dgh} j][\mathrm{fikl}] \\
& y_{13}=-[a b d f][\operatorname{cghi}][\mathrm{ejkl}] \quad y_{14}=-[\mathrm{abde}][\mathrm{cghj}][\mathrm{fikl}] \quad y_{15}=-[\mathrm{abcf}][\mathrm{dghj}][\mathrm{eikl}] \\
& y_{16}=[\mathrm{abdf}][\mathrm{cghj}][\mathrm{eikl}] \quad y_{17}=[\mathrm{abcg}][\mathrm{defi}][\mathrm{hjkl}] \quad y_{18}=-[\mathrm{abdg}][\mathrm{cefi}][\mathrm{hjkl}] \\
& y_{19}=-[a b c h][\operatorname{defi}][\mathrm{gjkl}] \quad y_{20}=-[\mathrm{abcg}][\operatorname{def} j][\mathrm{hikl}] \quad y_{21}=\text { [abdh][cefi][gjkl] } \\
& y_{22}=[\mathrm{abdg}][\mathrm{cefj}][\mathrm{hikl}] \quad y_{23}=[\mathrm{abch}][\mathrm{def} j][\mathrm{gikl}] \quad y_{24}=-[\mathrm{abdh}][\mathrm{cefj}][\mathrm{gikl}]
\end{aligned}
$$

\begin{tabular}{|c|c|c|c|}
\hline Rank & Class & \multicolumn{2}{|r|}{ Linear variety } \\
\hline 0 & empty set & \multicolumn{2}{|r|}{$\emptyset$} \\
\hline 1 & point & \multicolumn{2}{|r|}{ a line in the 3-D space } \\
\hline \multirow[t]{2}{*}{2} & \multirow[t]{2}{*}{ lines } & $(2 a)$ & a pair of skew lines \\
\hline & & (2b) & a flat pencil of lines \\
\hline \multirow[t]{4}{*}{3} & \multirow[t]{4}{*}{ planes } & $(3 a)$ & a regulus \\
\hline & & (3b) & $\begin{array}{l}\text { the union of two flat pencils having a line in common } \\
\text { but lying in distinct planes and with distinct centers }\end{array}$ \\
\hline & & $(3 \mathrm{c})$ & all lines through a point \\
\hline & & $(3 \mathrm{~d})$ & all lines in a plane \\
\hline \multirow[t]{4}{*}{4} & \multirow[t]{4}{*}{ congruences } & (4a) & elliptic congruence \\
\hline & & (4b) & hyperbolic congruence \\
\hline & & $(4 \mathrm{c})$ & parabolic congruence \\
\hline & & $(4 d)$ & degenerate congruence \\
\hline \multirow[t]{2}{*}{5} & \multirow[t]{2}{*}{ complexes } & (5a) & non singular complex; generated by five skew lines \\
\hline & & $(5 b)$ & singular complex; all the lines meeting one given line \\
\hline
\end{tabular}

A bracket [abcd] is null if and only if (iff) the projective points $a, b, c$ and $d$ are coplanar.

\subsection{Grassmann Geometry}

A general classification of linear varieties (sets of lines in the 3-dimensional projective space $\mathbb{P}_{3}$ ) can be found in [1, 9, 45] and is summarized in Table 1. Since a projective line can be either a finite line or a line at infinity, the foregoing classification does apply for a linear variety in which some lines at infinity may appear.

Table 1: Grassmann varieties [1,9,45].

\section{Constraint Analysis of 5-R $\underline{P} U R$ PMs}

\subsection{General design and kinematic properties}

Figures 1 and 2 provide respectively a schematic representation of a RPUR limb and a model of a 5-DOF PM that can be used to produce all three translational DOFs, plus two independent rotational DOFs (3T2R) of the end-effector. The mapping sequence between the desired orientation of the platform and angles $\theta$ and $\phi$ is the first rotation, $\theta$, about the $x$-axis followed by the second rotation, $\phi$, about the $y$-axis.

The input of the mechanism is provided by the five linear prismatic actuators. From the type synthesis presented in [25], the geometric characteristics associated with the components of each limb are as follows: The actuated prismatic joint of the $i$-th limb is directed along $2 \rho_{i} \| A_{i} B_{i}$ and line $\left(B_{i} C_{i}\right)$ is directed along $\mathbf{v}_{i}$. The first two revolute joints of the $i$-th limb have axes parallel to $\mathbf{e}_{1}$ and form a plane $\mathcal{P}_{i}$ while the axes of the last two ones are parallel to $\mathbf{e}_{2}$ and form a plane $\mathcal{V}_{i}$. It should be noted that the second and third revolute

\footnotetext{
${ }^{2}$ In what follows, operators “|"”, “ $\perp ”, ~ " \cap ”$ and “”” denote the parallelism, the orthogonality, the intersection and the coincidence of geometric entities, respectively.
} 


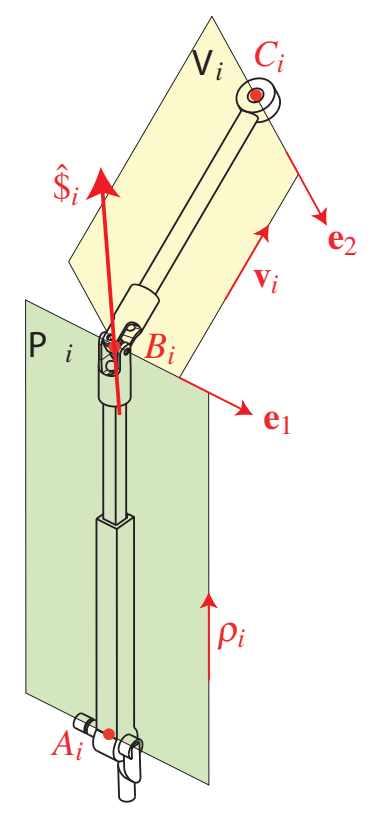

Figure 1: Schematic representation of a RPUR limb.

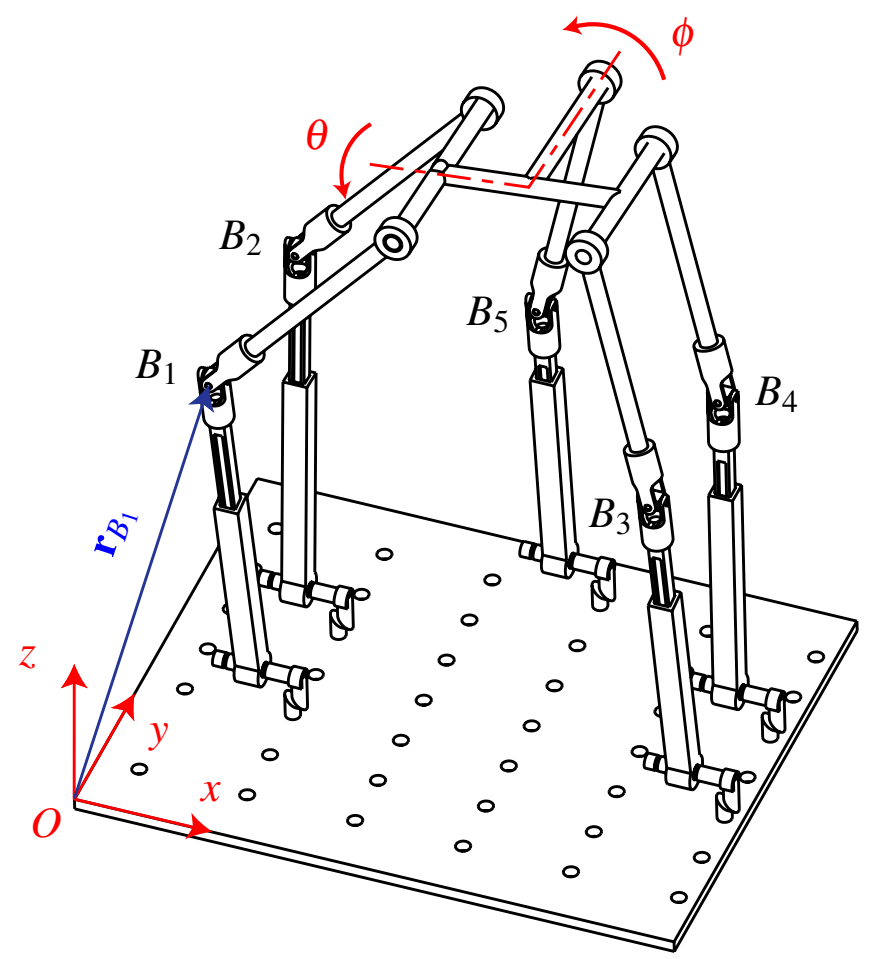

Figure 2: Schematic representation of a 5-R $\underline{P} U R$ PM.

joints in each limb are arranged with perpendicular axes intersecting at point $B_{i}$ and are thus assimilated to a $U$ (Universal) joint. This mechanical simplification leads to a 5-RPUR type mechanism. Further results regarding the kinematic properties such as the solution of the IKP, the FKP and the determination of the constant orientation workspace can be found in [18, 19, 31-34]. It should be noted that the origin of this research work is based on the results obtained for their FKP, with 1680 finite solutions [18], and the constant-orientation workspace where Bohemian domes [46] come up for the vertex space [33]. 


\subsubsection{Constraint wrench system}

Each limb of the PM applies a constraint wrench, which is a pure moment (infinite-pitch wrench) $\hat{\$}_{c}=\left(\mathbf{0} ; \mathbf{e}_{1} \times\right.$ $\mathbf{e}_{2}$ ) being reciprocal to the five twists associated with the limb's kinematic joints $R, \underline{P}, U$ and $R$. Consequently, all the limbs of this 5-DOF PM apply the same constraint wrench implying that symmetric 5-DOF PMs are highly overconstrained. Therefore, the constraint wrench system $W_{c}$ of the PM is a 1-system given by:

$$
\mathrm{W}_{c}=\operatorname{span}\left(\hat{\$}_{c}\right) \quad, \quad \hat{\$}_{c}=\left(\mathbf{0} ; \mathbf{e}_{1} \times \mathbf{e}_{2}\right)
$$

The moving platform cannot rotate about any axis not normal to $\mathbf{e}_{3}=\mathbf{e}_{1} \times \mathbf{e}_{2}$. It has three independent translations and two independent rotations whose axes are orthogonal to $\mathbf{e}_{3}$.

Emerging here is the notion of platform singularity (constraint singularity [7]). From Eq. (2), it follows that the order of the constraint wrench system for this class of 5-DOF PMs cannot be lower than one, since $\mathbf{e}_{1}$ and $\mathbf{e}_{2}$ are two fixed independent directions, which lets aside the platform singularity from the remainder of the singularity analysis [25].

\subsubsection{Actuation wrench system}

By locking the actuator in the $i$-th limb, an additional constraint appears, which is called the actuation wrench of the limb. It is a pure force reciprocal to the limb's four revolute joints. Thus, the axis of this wrench is the intersection line of planes $\mathcal{P}_{i}$ and $\mathcal{V}_{i}$ and passes necessarily through point $B_{i}$. Consequently, each limb $l_{i}$ of the mechanism applies one actuation force $\hat{\$}_{i}, i=1, \ldots, 5$, of direction $\mathbf{s}_{i}$ and of axis $A_{i}=\mathcal{P}_{i} \cap \mathcal{V}_{i}$ as depicted in Fig. 1. Plane $\mathcal{P}_{i}$ has $\left(\mathbf{e}_{1} \times \rho_{i}\right)$ as normal vector while plane $\mathcal{V}_{i}$ has $\left(\mathbf{e}_{2} \times \mathbf{v}_{i}\right)$ as normal one. The actuation force of the $i$-th limb is expressed as:

$$
\hat{\$}_{i}=\left(\mathbf{s}_{i} ; \mathbf{r}_{B_{i}} \times \mathbf{s}_{i}\right), \mathbf{s}_{i}=\left(\mathbf{e}_{1} \times \rho_{i}\right) \times\left(\mathbf{e}_{2} \times \mathbf{v}_{i}\right)
$$

where $\mathbf{r}_{B_{i}}$ is the position vector of point $B_{i}$ with respect to the reference frame $(O, x, y, z)$.

\subsubsection{Jacobian matrix and overall wrench system}

The rows of the Jacobian matrix $\mathbf{J}$ of the 5-RPUR PM can be expressed as the six unit screws $\hat{\$}_{1}, \hat{\$}_{2}, \hat{\$}_{3}, \hat{\$}_{4}, \hat{\$}_{5}$ and $\hat{\$}_{c}$ that correspond to the Plücker coordinate vectors of six projective lines. The overall wrench system of the PM is given by:

$$
\mathrm{W}=\operatorname{span}\left(\hat{\$}_{1}, \hat{\$}_{2}, \hat{\$}_{3}, \hat{\$}_{4}, \hat{\$}_{5}, \hat{\$}_{c}\right)
$$

In a non singular configuration, $\mathrm{W}$ is a 6 -system and the six screws are linearly independent. Singular configurations of the mechanism correspond to configurations in which these six screws become linearly dependent and span a $(n<6)$-system.

\subsection{Simplified designs}

The PMs under study in this paper are the results of a recent study [19] where the main purpose was to obtain a family of simplified designs whose FKP admits either a closed-form solution or a univariate expression. This issue, i.e., having a simple FKP, is a definite asset in the design of PMs, which makes the control of the PM easier. Two among the nine simplified designs presented in [19], named $\mathcal{A} A$ and $\mathcal{B} B$, are considered as case studies to apply the GCA. Then, the results are compared with those obtained in [32] using GG.

\subsubsection{The $\mathcal{A} A$ mechanism}

The $\mathcal{A} \mathcal{A}$ mechanism shown in Fig. [3 is obtained from the general architecture with $B_{1} \equiv B_{2} \equiv B_{12}, B_{3} \equiv$ $B_{4} \equiv B_{34}, C_{1} \equiv C_{2} \equiv C_{12}$ and $C_{3} \equiv C_{4} \equiv C_{34}$. In turn, plane $\mathcal{V}_{1} \equiv \mathcal{V}_{2} \equiv \mathcal{V}_{12}$ is defined as the plane containing $B_{12}, C_{12}$ and $\mathbf{e}_{2}$ whereas vector $\mathbf{v}_{1} \equiv \mathbf{v}_{2} \equiv \mathbf{v}_{12}$ lies in plane $\mathcal{V}_{12}$ and is orthogonal to $\mathbf{e}_{2}$. Similarly for $\mathcal{V}_{34}$ and $\mathbf{v}_{34}$.

Let $A_{i}$ denote the axis of screw $\hat{\$}_{i}$ of direction $\mathbf{s}_{i}$. We have $A_{1}=\mathcal{P}_{1} \cap \mathcal{V}_{12}$ and $A_{2}=\mathcal{P}_{2} \cap \mathcal{V}_{12}$. Moreover, $A_{1}$ and $A_{2}$ intersect at point $B_{12}$ which belongs to the three planes $\mathcal{P}_{1}, \mathcal{P}_{2}$ and $\mathcal{V}_{12}$. Screws $\hat{\$}_{1}$ and $\hat{\$}_{2}$ are given by:

\footnotetext{
${ }^{3}$ For the $i$-th limb, point $B_{i}$ is defined as the intersection point of the second and the third revolute joint axes.
} 


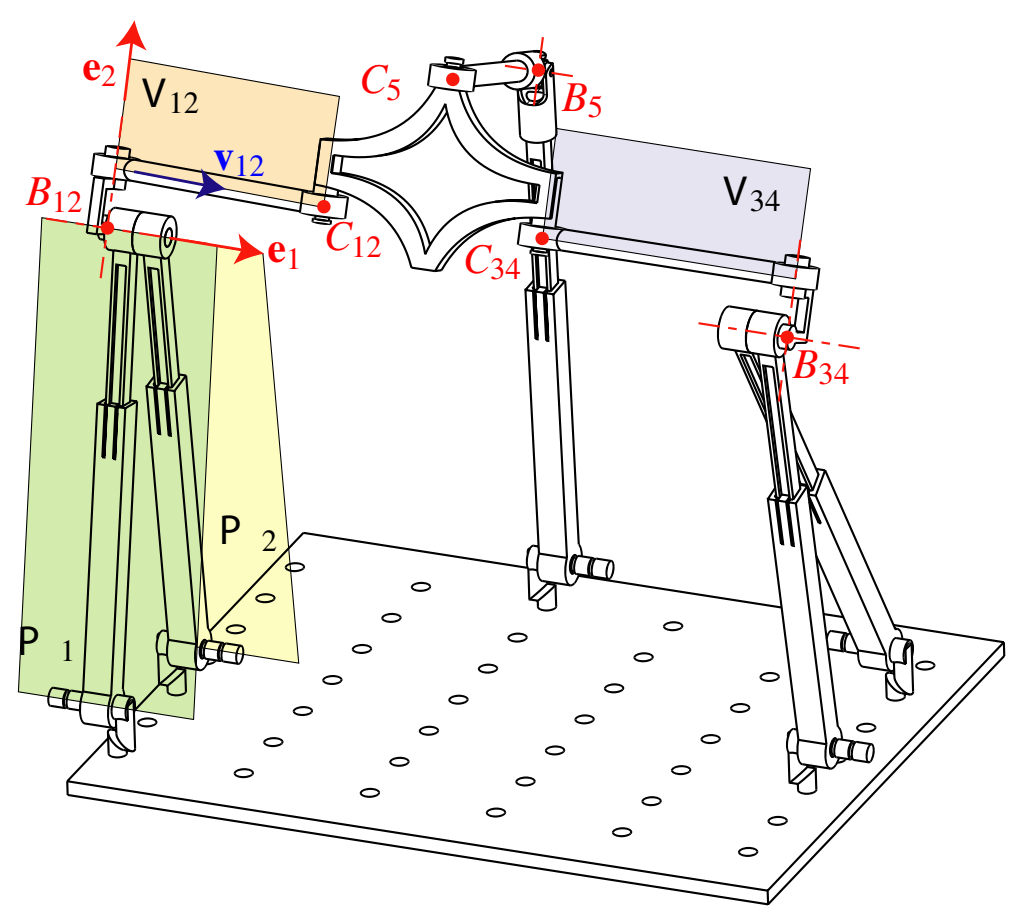

Figure 3: Schematic representation of the $\mathcal{A A}$ mechanism.

- $\hat{\$}_{1}=\left(\mathbf{s}_{1} ; \mathbf{r}_{B_{12}} \times \mathbf{s}_{1}\right)$, where $\mathbf{s}_{1}=\left(\mathbf{e}_{1} \times \rho_{1}\right) \times\left(\mathbf{e}_{2} \times \mathbf{v}_{12}\right)$;

- $\hat{\$}_{2}=\left(\mathbf{s}_{2} ; \mathbf{r}_{B_{12}} \times \mathbf{s}_{2}\right)$, where $\mathbf{s}_{2}=\left(\mathbf{e}_{1} \times \rho_{2}\right) \times\left(\mathbf{e}_{2} \times \mathbf{v}_{12}\right)$.

Now let us consider screws $\hat{\$}_{3}, \hat{\$}_{4}$ and $\hat{\$}_{5}$. We have $A_{3}=\mathcal{P}_{3} \cap \mathcal{V}_{34}, A_{4}=\mathcal{P}_{4} \cap \mathcal{V}_{34}$ and $A_{5}=\mathcal{P}_{5} \cap \mathcal{V}_{5}$. Moreover, $A_{3}$ and $A_{4}$ intersect at point $B_{34}$. Thus, screws $\hat{\$}_{3}, \hat{\$}_{4}$ and $\hat{\$}_{5}$ are given by:

- $\hat{\$}_{3}=\left(\mathbf{s}_{3} ; \mathbf{r}_{B_{34}} \times \mathbf{s}_{3}\right)$, where $\mathbf{s}_{3}=\left(\mathbf{e}_{1} \times \rho_{3}\right) \times\left(\mathbf{e}_{2} \times \mathbf{v}_{34}\right)$;

- $\hat{\$}_{4}=\left(\mathbf{s}_{4} ; \mathbf{r}_{B_{34}} \times \mathbf{s}_{4}\right)$, where $\mathbf{s}_{4}=\left(\mathbf{e}_{1} \times \rho_{4}\right) \times\left(\mathbf{e}_{2} \times \mathbf{v}_{34}\right)$;

- $\hat{\$}_{5}=\left(\mathbf{s}_{5} ; \mathbf{r}_{B_{5}} \times \mathbf{s}_{5}\right)$, where $\mathbf{s}_{5}=\left(\mathbf{e}_{1} \times \rho_{5}\right) \times\left(\mathbf{e}_{2} \times \mathbf{v}_{5}\right)$.

Plane $\mathcal{V}_{12}$ contains axes $A_{1}$ and $A_{2}$ of the two screws $\hat{\$}_{1}$ and $\hat{\$}_{2}$. Likewise, plane $\mathcal{V}_{34}$ contains axes $A_{3}$ and $A_{4}$ of $\hat{\$}_{3}$ and $\hat{\$}_{4}$.

\subsubsection{The $\mathcal{B} B$ mechanism}

For the $\mathcal{B B}$ mechanism shown in Fig. 4, we have $A_{1} \equiv A_{2} \equiv A_{12}, A_{3} \equiv A_{4} \equiv A_{34}$ and $C_{1} C_{2}\left\|C_{3} C_{4}\right\| \mathbf{e}_{2}$. The mechanism is assembled such that $B_{1} C_{1}\left\|B_{2} C_{2}\right\| \mathbf{v}_{12}, B_{3} C_{3}\left\|B_{4} C_{4}\right\| \mathbf{v}_{34}$. Thus, $\mathcal{V}_{1} \equiv \mathcal{V}_{2} \equiv \mathcal{V}_{12}$, $\mathcal{V}_{3} \equiv \mathcal{V}_{4} \equiv \mathcal{V}_{34}$ and $B_{1} B_{2}\left\|B_{3} B_{4}\right\| \mathbf{e}_{2}$. In this paper, the singularity analysis is performed for this working mode of the mechanism. It is noteworthy that the $\mathcal{B B}$ mechanism has other working modes for which plane $\mathcal{V}_{12}\left(\mathcal{V}_{34}\right.$, respectively) may not exist. The working mode change appears when the robot goes through a limb singularity. For example, if $\mathbf{v}_{4} \| \mathbf{e}_{1}$, the mechanism will be able to switch into another working mode in which $B_{3} C_{3}$ is no longer parallel to $B_{4} C_{4}$. However, it is possible to prevent this mode change by imposing some limits on the actuated prismatic joints.

The axis $\mathrm{A}_{i}$ of screw $\hat{\$}_{i},(i=1, \ldots, 5)$, passes through point $B_{i}$. Screws $\hat{\$}_{1}, \hat{\$}_{2}, \hat{\$}_{3}, \hat{\$}_{4}, \hat{\$}_{5}$ and $\hat{\$}_{c}$ take the following expressions:

- $\hat{\$}_{1}=\left(\mathbf{s}_{1} ; \mathbf{r}_{B_{1}} \times \mathbf{s}_{1}\right)$, where $\mathbf{s}_{1}=\left(\mathbf{e}_{1} \times \rho_{1}\right) \times\left(\mathbf{e}_{2} \times \mathbf{v}_{12}\right)$;

- $\hat{\$}_{2}=\left(\mathbf{s}_{2} ; \mathbf{r}_{B_{2}} \times \mathbf{s}_{2}\right)$, where $\mathbf{s}_{2}=\left(\mathbf{e}_{1} \times \rho_{2}\right) \times\left(\mathbf{e}_{2} \times \mathbf{v}_{12}\right)$;

- $\hat{\$}_{3}=\left(\mathbf{s}_{3} ; \mathbf{r}_{B_{3}} \times \mathbf{s}_{3}\right)$, where $\mathbf{s}_{3}=\left(\mathbf{e}_{1} \times \rho_{3}\right) \times\left(\mathbf{e}_{2} \times \mathbf{v}_{34}\right)$; 


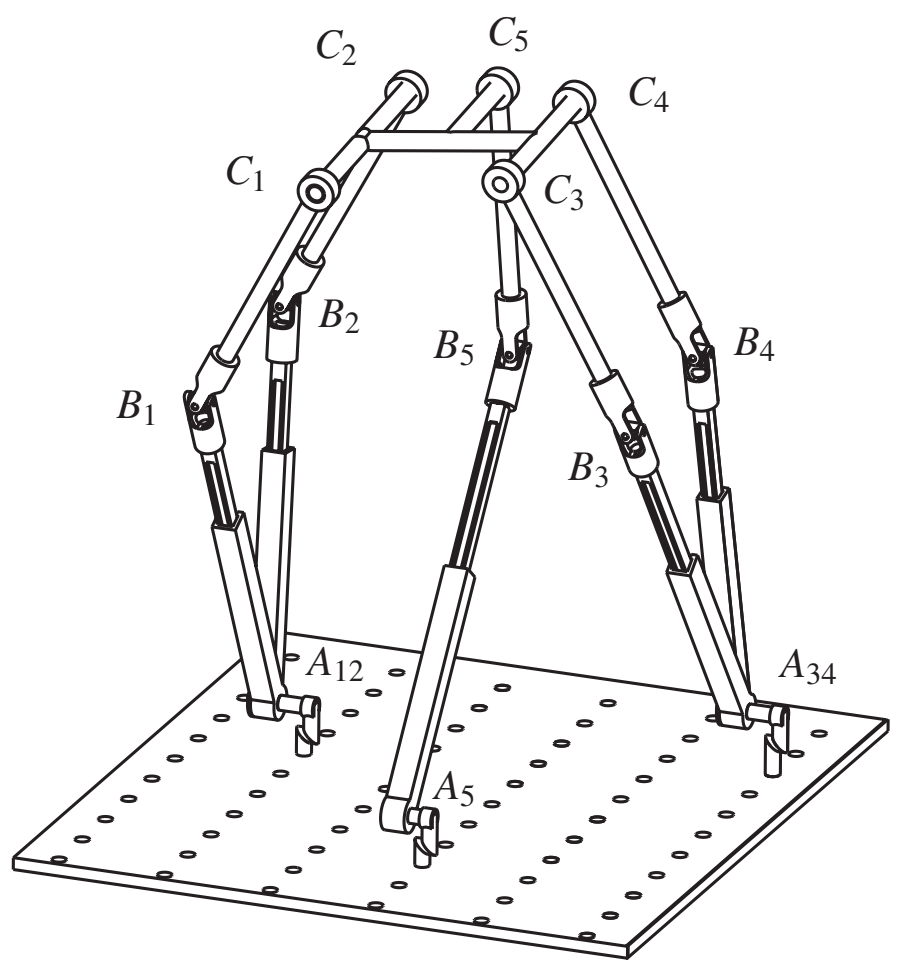

Figure 4: Schematic representation of the $\mathcal{B B}$ mechanism.

- $\hat{\$}_{4}=\left(\mathbf{s}_{4} ; \mathbf{r}_{B_{4}} \times \mathbf{s}_{4}\right)$, where $\mathbf{s}_{4}=\left(\mathbf{e}_{1} \times \rho_{4}\right) \times\left(\mathbf{e}_{2} \times \mathbf{v}_{34}\right)$;

- $\hat{\$}_{5}=\left(\mathbf{s}_{5} ; \mathbf{r}_{B_{5}} \times \mathbf{s}_{5}\right)$, where $\mathbf{s}_{5}=\left(\mathbf{e}_{1} \times \rho_{5}\right) \times\left(\mathbf{e}_{2} \times \mathbf{v}_{5}\right)$;

- $\hat{\$}_{c}=\left(\mathbf{0} ; \mathbf{e}_{3}\right)$.

Let us consider axes $A_{3}$ and $A_{4}$ of screws $\hat{\$}_{3}$ and $\hat{\$}_{4}$, respectively. As mentioned previously, these axes pass through points $B_{3}$ and $B_{4}$, respectively. Moreover, we have $A_{3}=\mathcal{P}_{3} \cap \mathcal{V}_{34}$ and $A_{4}=\mathcal{P}_{4} \cap \mathcal{V}_{34}$. Thus, plane $\mathcal{V}_{34}$ contains axes $A_{3}$ and $A_{4}$ that intersect at point $B_{34}$. Obviously, this point lies in the three planes $\mathcal{P}_{3}, \mathcal{P}_{4}$ and $\mathcal{V}_{34}$. As shown in Fig 5 , the intersection line of $\mathcal{P}_{3}$ and $\mathcal{P}_{4}$ coincides with the axis of the $\mathrm{R}$-joint of direction $\mathbf{e}_{1}$, passing through point $A_{34}$ and attached to the fixed base. Therefore, point $B_{34}$ is the intersection point of this axis and plane $\mathcal{V}_{34}$. Likewise, axes $A_{1}$ and $A_{2}$ intersect in plane $\mathcal{V}_{12}$ at point $B_{12}$ that also belongs to the line passing through point $A_{12}$ and directed along $\mathbf{e}_{1}$.

\section{Singularity Analysis of the $\mathcal{A} \mathcal{A}$ mechanism}

\subsection{Superbracket of the $\mathcal{A} \mathcal{A}$ mechanism}

A spanning system of the overall wrench system of the $\mathcal{A A}$ mechanism is composed of one constraint moment $\hat{\$}_{c}=\left(\mathbf{0} ; \mathbf{e}_{3}\right)$, and five actuation forces $\hat{\$}_{i}=\left(\mathbf{s}_{i} ; \mathbf{r}_{B_{i}} \times \mathbf{s}_{i}\right),(i=1, \ldots, 5)$ as obtained in Sec. 3.2.1. These wrenches are represented with one line at infinity and five finite lines in $\mathbb{P}_{3}$. In order to obtain the twelve points of the superbracket corresponding to the mechanism under study, we must select twelve points on these six projective lines, i.e. two points on each line. The extensor of a line at infinity is the join of two extensors associated with two distinct points at infinity belonging to this line. The extensor of a finite line is the join of two extensors associated either with two distinct finite points or with one finite point and one point at infinity, since any finite line has one point at infinity defining its direction.

A wrench graph representing the projective lines associated with the wrenches of the $\mathcal{A A}$ mechanism is given in Fig. 6. Let $\underline{\mathrm{e}}_{1}, \underline{\mathrm{e}}_{2}$ and $\underline{\mathrm{e}}_{3}$ be the extensors associated with the points at infinity in the directions $\mathbf{e}_{1}, \mathbf{e}_{2}$ and $\mathbf{e}_{3}$, respectively. Thus, $\underline{\mathrm{e}}_{1}=\left(\mathbf{e}_{1} ; 0\right), \underline{\mathrm{e}}_{2}=\left(\mathbf{e}_{2} ; 0\right)$ and $\hat{\phi}_{c}=\underline{\mathrm{e}}_{1} \underline{\mathrm{e}}_{2}=\left(\mathbf{0} ; \mathbf{e}_{1} \times \mathbf{e}_{2}\right)=\left(\mathbf{0} ; \mathbf{e}_{3}\right)$. 


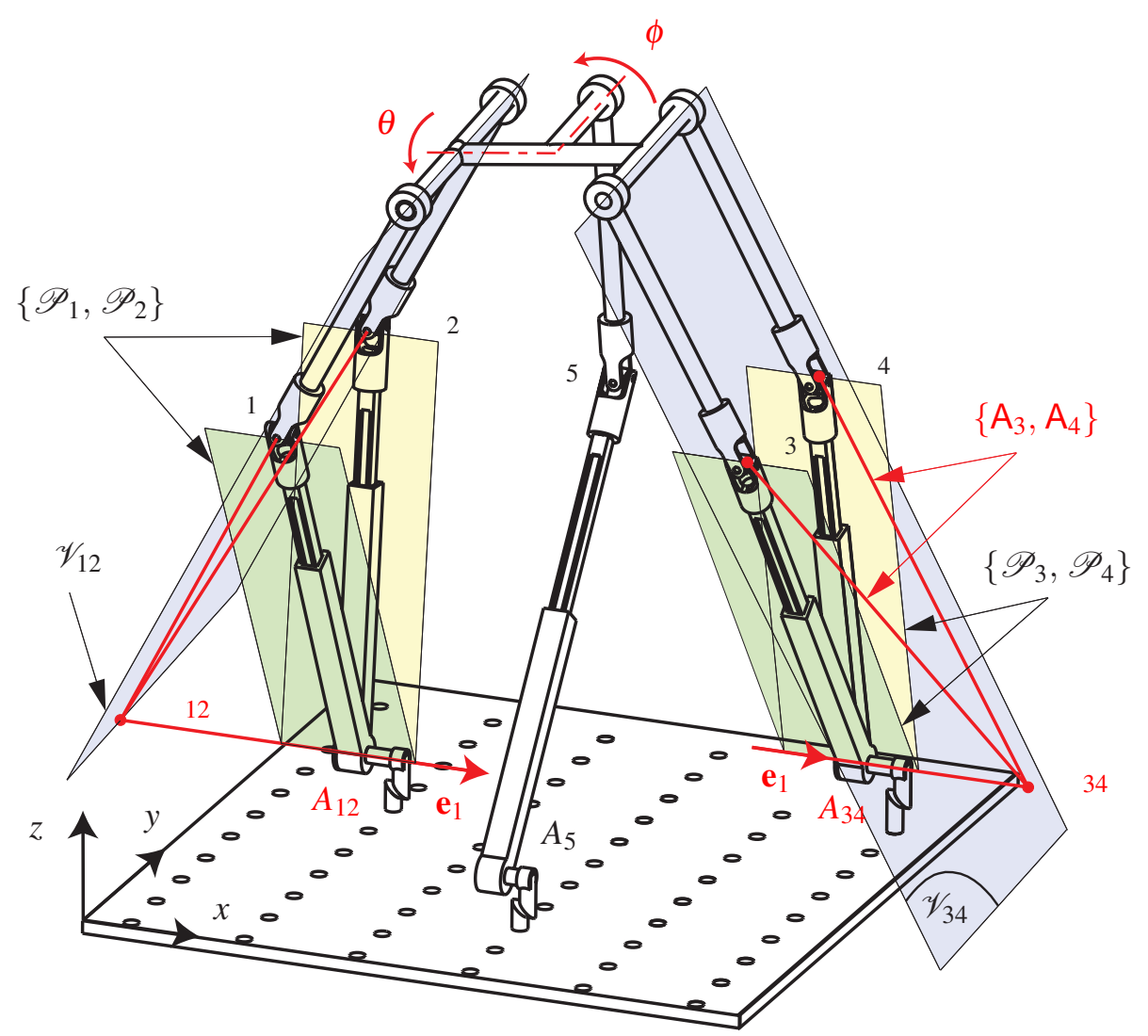

Figure 5: Planes $\mathcal{P}_{i}$ and $\mathcal{V}_{i}$ for the $\mathcal{B B}$ mechanism.

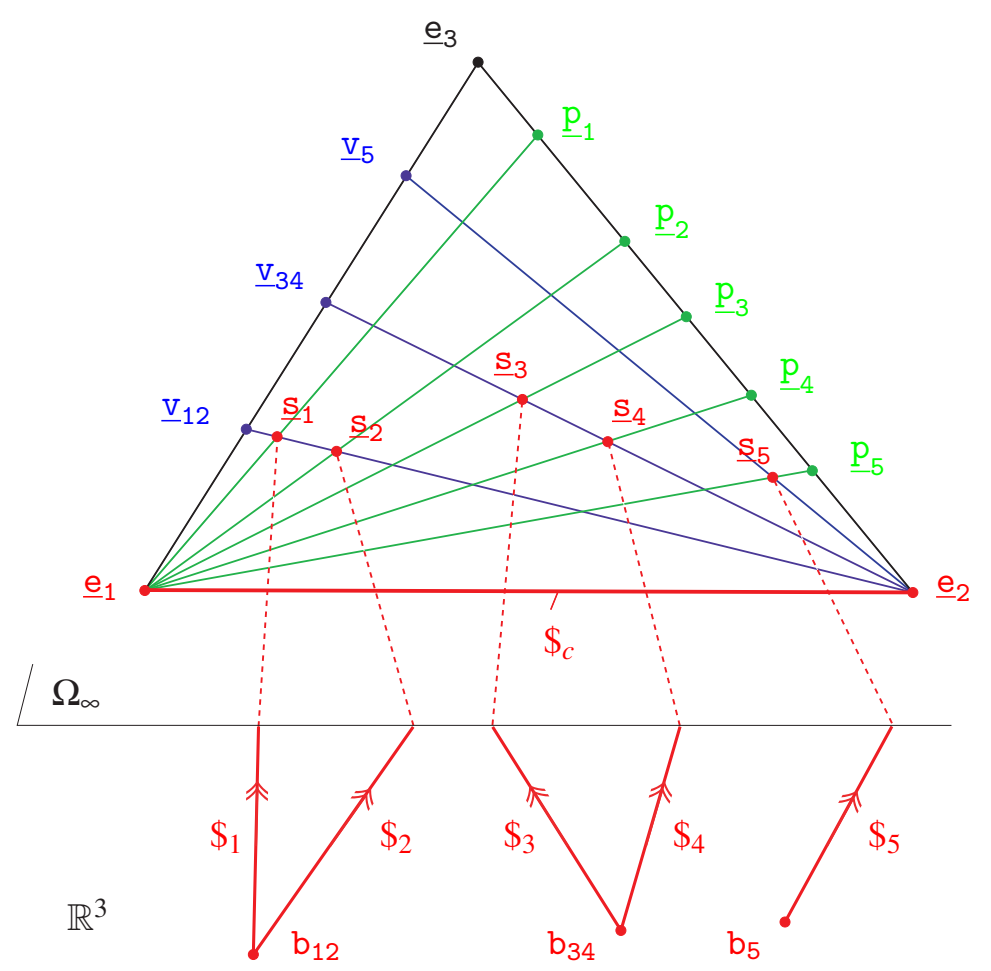

Figure 6: Wrench graph of the $\mathcal{A} \mathcal{A}$ mechanism in $\mathbb{P}_{3}$.

Let $\underline{\mathrm{p}}_{i},(i=1, \ldots, 5)$, be the extensor associated with the point at infinity in the direction $\rho_{i}$. Thus, $\underline{\mathrm{p}}_{i}=\left(\underline{\rho}_{i} ; 0\right)$. The line at infinity $\underline{\mathrm{e}}_{2} \underline{\mathrm{e}}_{3}=\left(\mathbf{0} ; \mathbf{e}_{2} \times \mathbf{e}_{3}\right)=\left(\mathbf{0} ; \mathbf{e}_{1}\right)$ passes through all points at infinity of $\underline{\mathrm{p}}_{i}$ directions orthogonal to $\mathbf{e}_{1}$. Since $\rho_{i},(i=1, \ldots, 5)$, are orthogonal to $\mathbf{e}_{1}$, points $\underline{\mathrm{p}}_{i}$ belong to $\underline{\mathrm{e}}_{2} \underline{\mathrm{e}}_{3}$.

Let $\underline{\mathrm{v}}_{12}, \underline{\mathrm{v}}_{34}$ and $\underline{\mathrm{v}}_{5}$ be the extensors associated with the points at infinity in the directions $\mathbf{v}_{12}, \mathbf{v}_{34}$ and $\mathbf{v}_{5}$, 
respectively. Thus, $\underline{\mathrm{v}}_{12}=\left(\mathbf{v}_{12} ; 0\right), \underline{\mathrm{v}}_{34}=\left(\mathbf{v}_{34} ; 0\right)$ and $\underline{\mathrm{v}}_{5}=\left(\mathbf{v}_{5} ; 0\right)$. Since $\mathbf{v}_{12}, \mathbf{v}_{34}$ and $\mathbf{v}_{5}$ are orthogonal to $\mathbf{e}_{2}$, points $\underline{\mathrm{v}}_{12}, \underline{\mathrm{v}}_{34}$ and $\underline{\mathrm{v}}_{5}$ belong to $\underline{\mathrm{e}}_{1} \underline{\mathrm{e}}_{3}=\left(\mathbf{0} ; \mathbf{e}_{1} \times \mathbf{e}_{3}\right)=\left(\mathbf{0} ; \mathbf{e}_{2}\right)$.

The line at infinity $\mathcal{L}_{P_{i}}$ of plane $\mathcal{P}_{i}$ passes through $\underline{\mathrm{p}}_{i}$ and $\underline{\mathrm{e}}_{1}$, i.e., $\mathcal{L}_{P_{i}}=\underline{\mathrm{e}}_{1} \underline{\mathrm{p}}_{i}=\left(\mathbf{0} ; \mathbf{e}_{1} \times \rho_{i}\right),(i=1, \ldots, 5)$. Similarly, the lines at infinity of planes $\mathcal{V}_{12}, \mathcal{V}_{34}$ and $\overline{\mathcal{V}}_{5}^{i}$ are $\overline{\mathcal{L}}_{V_{12}}=\underline{\mathrm{e}}_{2} \underline{\mathrm{v}}_{12}=\left(\mathbf{0} ; \mathbf{e}_{2} \times \mathbf{v}_{12}\right), \mathcal{L}_{V_{34}}=\underline{\mathrm{e}}_{2} \underline{\mathrm{v}}_{34}=$ $\left(\mathbf{0} ; \mathbf{e}_{2} \times \mathbf{v}_{34}\right)$ and $\mathcal{L}_{V_{5}}=\underline{\mathrm{e}}_{2} \underline{\mathrm{v}}_{5}=\left(\mathbf{0} ; \mathbf{e}_{2} \times \mathbf{v}_{5}\right)$.

Let $\mathrm{b}_{12}, \mathrm{~b}_{34}$ and $\mathrm{b}_{5}$ be the extensors associated with points $B_{12}, B_{34}$ and $B_{5}$, respectively, and let $\underline{\mathrm{s}}_{i}$, $(i=1, \ldots, 5)$ represent the point at infinity of axis $\mathrm{A}_{i}$. Since $\mathcal{P}_{1} \cap \mathcal{V}_{12}=\mathrm{A}_{1}$, the lines at infinity of $\mathcal{P}_{1}$ and $\mathcal{V}_{12}$ intersect at point $\underline{\mathrm{s}}_{1}$. Accordingly,

$$
\underline{\mathrm{s}}_{1}=\mathcal{L}_{P_{1}} \cap \mathcal{L}_{V_{12}}=\underline{\mathrm{e}}_{1} \underline{\mathrm{p}}_{1} \wedge \underline{\mathrm{e}}_{2} \underline{\mathrm{v}}_{12}
$$

Likewise,

$$
\begin{aligned}
& \underline{\mathrm{s}}_{2}=\mathcal{L}_{P_{2}} \cap \mathcal{L}_{V_{12}}=\underline{\mathrm{e}}_{1} \underline{\mathrm{p}}_{2} \wedge \underline{\mathrm{e}}_{2} \underline{\mathrm{v}}_{12} \\
& \underline{\mathrm{s}}_{3}=\mathcal{L}_{P_{3}} \cap \mathcal{L}_{V_{34}}=\underline{\mathrm{e}}_{1} \underline{\mathrm{p}}_{3} \wedge \underline{\mathrm{e}}_{2} \underline{\mathrm{v}}_{34} \\
& \underline{\mathrm{s}}_{4}=\mathcal{L}_{P_{4}} \cap \mathcal{L}_{V_{34}}=\underline{\mathrm{e}}_{1} \underline{\mathrm{p}}_{4} \wedge \underline{\mathrm{e}}_{2} \underline{\mathrm{v}}_{34} \\
& \underline{\mathrm{s}}_{5}=\mathcal{L}_{P_{5}} \cap \mathcal{L}_{V_{5}}=\underline{\mathrm{e}}_{1} \underline{\mathrm{p}}_{5} \wedge \underline{\mathrm{e}}_{2} \underline{\mathrm{v}}_{5}
\end{aligned}
$$

As a consequence, $\hat{\$}_{1}=\mathrm{b}_{12} \underline{\mathrm{s}}_{1}, \hat{\$}_{2}=\mathrm{b}_{12 \underline{\mathrm{s}}_{2}}, \hat{\mathrm{\$}}_{3}=\mathrm{b}_{34} \underline{\mathrm{s}}_{3}, \hat{\$}_{4}=\mathrm{b}_{4} \underline{\mathrm{s}}_{4}, \hat{\$}_{5}=\mathrm{b}_{5} \underline{\mathrm{s}}_{5}$ and $\hat{\$}_{c}=\underline{\mathrm{e}}_{1} \underline{\mathrm{e}}_{2}$. The superbracket of the $\mathcal{A} \mathcal{A}$ mechanism is expressed as follows:

$$
S_{A A}=\left[b_{12} \underline{\mathbf{s}}_{1}, \mathrm{~b}_{12} \underline{\mathbf{s}}_{2}, \mathrm{~b}_{5} \underline{\mathbf{s}}_{5}, \underline{\mathrm{e}}_{1} \underline{\mathrm{e}}_{2}, \mathrm{~b}_{34} \underline{\mathrm{s}}_{3}, \mathrm{~b}_{34} \underline{\mathrm{s}}_{4}\right]
$$

\subsection{Superbracket decomposition of the $\mathcal{A} \mathcal{A}$ mechanism}

From Eq. (1), the superbracket can be developed into a linear combination of 24 monomials. Since the bracket of any four coplanar projective points is null, 20 among the 24 monomials of $S_{A A}$ are null. Therefore, $S_{A A}$ takes the following simplified expression:

$$
\begin{aligned}
& S_{A A}=-\left[b_{12} b_{5} \underline{\mathbf{s}}_{5} b_{34}\right]\left[b_{12} \underline{\mathbf{s}}_{1} \underline{\mathbf{s}}_{2} \underline{\mathrm{e}}_{1}\right]\left[\underline{e}_{2} \underline{\mathbf{s}}_{3} b_{34} \underline{\mathbf{s}}_{4}\right]+\left[b_{12} b_{5} \underline{\mathbf{s}}_{5} b_{34}\right]\left[b_{12} \underline{\mathbf{s}}_{1} \underline{\mathbf{s}}_{2} \underline{\mathrm{e}}_{2}\right]\left[\underline{e}_{1} \underline{\mathbf{s}}_{3} \mathrm{~b}_{34} \underline{\mathbf{s}}_{4}\right] \\
& +\left[b_{12} \underline{e}_{1} \underline{e}_{2} b_{34}\right]\left[b_{12} \underline{s}_{1} \underline{s}_{2} b_{5}\right]\left[\underline{s}_{5} \underline{s}_{3} b_{34} \underline{s}_{4}\right]-\left[b_{12} \underline{e}_{1} \underline{e}_{2} b_{34}\right]\left[b_{12} \underline{s}_{1} \underline{s}_{2} \underline{s}_{5}\right]\left[b_{5} \underline{\mathbf{s}}_{3} b_{34} \underline{\mathbf{s}}_{4}\right] \\
& S_{A A}=-\left[b_{12} b_{5} \underline{\mathbf{s}}_{5} b_{34}\right]\left(\left[b_{12} \underline{\mathbf{s}}_{1} \underline{\mathbf{s}}_{2} \underline{\dot{e}}_{1}\right]\left[\underline{e}_{2} \underline{\mathbf{s}}_{3} b_{34} \underline{\mathbf{s}}_{4}\right]\right)+\left[b_{12} \underline{\mathrm{e}}_{1} \underline{e}_{2} b_{34}\right]\left(\left[b_{12} \underline{\mathbf{s}}_{1} \underline{\mathbf{s}}_{2} \dot{b}_{5}\right]\left[\underline{\dot{s}}_{5} \underline{\mathbf{s}}_{3} b_{34} \underline{\mathbf{s}}_{4}\right]\right)
\end{aligned}
$$

where the dotted letters stand for permuted elements as explained in [11,42]. The previous expression is composed of two main terms $X$ and $Y$, namely,

$$
\begin{aligned}
& \left.X=\left[\mathrm{b}_{12} \mathrm{~b}_{5} \underline{\mathrm{s}}_{5} \mathrm{~b}_{34}\right]\left(\left[\mathrm{b}_{12} \underline{\mathrm{s}}_{1} \underline{\mathrm{s}}_{2} \underline{\dot{e}}_{1}\right] \underline{\dot{e}}_{2} \underline{\mathrm{s}}_{3} \mathrm{~b}_{34} \underline{\mathrm{s}}_{4}\right]\right) \\
& Y=\left[\mathrm{b}_{12} \underline{\mathrm{e}}_{1} \underline{\mathrm{e}}_{2} \mathrm{~b}_{34}\right]\left(\left[\mathrm{b}_{12} \underline{\mathrm{s}}_{1} \underline{\mathrm{s}}_{2} \dot{\mathrm{b}}_{5}\right]\left[\underline{\mathbf{s}}_{5} \underline{\mathrm{s}}_{3} \mathrm{~b}_{34} \underline{\mathrm{s}}_{4}\right]\right)
\end{aligned}
$$

The first term $X$ is equal to zero when:

1. $\left[b_{12} b_{5} \underline{\mathbf{s}}_{5} b_{34}\right]=0 \Rightarrow$ points $b_{12}, b_{5}, b_{34}$ and $\underline{s}_{5}$ are coplanar;

2. $\left(b_{12} \underline{s}_{1} \underline{\mathbf{s}}_{2}\right) \wedge\left(\underline{s}_{3} b_{34} \underline{\mathbf{s}}_{4}\right) \wedge\left(\underline{e}_{1} \underline{e}_{2}\right)=0 \Rightarrow$ the projective line $\left(\underline{e}_{1} \underline{e}_{2}\right)$ intersects the intersection line of planes $\left(\mathrm{b}_{12} \underline{\mathbf{S}}_{1} \underline{\mathbf{S}}_{2}\right)$ and $\left(\underline{\mathbf{s}}_{3} \mathrm{~b}_{34} \underline{\mathbf{S}}_{4}\right)$. Plane $\left(\mathrm{b}_{12} \underline{\mathbf{s}}_{1} \underline{\mathbf{s}}_{2}\right)$ is the plane containing the finite point $B_{12}$ and the two points at infinity $\underline{\mathbf{s}}_{1}$ and $\underline{\mathbf{s}}_{2}$ of directions $\mathbf{s}_{1}$ and $\mathbf{s}_{2}$, respectively. From Sec. 3.2.1, since plane $\mathcal{V}_{12}$ (respectively $\mathcal{V}_{34}$ ) contains axes $A_{1}$ and $A_{2}$ (respectively $A_{3}$ and $A_{4}$ ) of the two screws $\hat{\$}_{1}$ and $\hat{\$}_{2}$ (respectively $\hat{\$}_{3}$ and $\left.\hat{\$}_{4}\right)$, we conclude that $\left(\mathrm{b}_{12} \underline{\mathbf{S}}_{1} \underline{\mathrm{S}}_{2}\right) \equiv \mathcal{V}_{12}\left(\right.$ respectively $\left.\left(\underline{\mathrm{s}}_{3} \mathrm{~b}_{34} \underline{\underline{S}}_{4}\right) \equiv \mathcal{V}_{34}\right)$. Therefore,

$$
\left(b_{12} \underline{s}_{1} \underline{s}_{2}\right) \wedge\left(\underline{s}_{3} b_{34} \underline{s}_{4}\right) \equiv\left(\mathcal{V}_{12} \cap \mathcal{V}_{34}\right)
$$

Moreover, the lines at infinity of planes $\mathcal{V}_{12}$ and $\mathcal{V}_{34}$ intersect at point $\underline{\mathbf{e}}_{2}$. Thus, $\left(\mathrm{b}_{12} \underline{\mathbf{s}}_{1} \underline{\mathbf{s}}_{2}\right) \wedge\left(\underline{\mathbf{s}}_{3} b_{34} \underline{\mathbf{s}}_{4}\right)$ is a finite line of direction $\mathbf{e}_{2}$, which always intersects the line at infinity $\left(\underline{e}_{1} \underline{e}_{2}\right)$ at point $\left.\underline{e}_{2}\right]$.

\footnotetext{
${ }^{4}$ More generally, the condition $\left(\mathrm{b}_{12} \underline{\underline{s}}_{1} \underline{\underline{s}}_{2}\right) \wedge\left(\underline{s}_{3} b_{34} \underline{s}_{4}\right) \wedge\left(\underline{e}_{1} \underline{e}_{2}\right)=0$ is verified when line $\left(\mathcal{V}_{12} \cap \mathcal{V}_{34}\right)$ lies in a plane having $\mathbf{e}_{3}$ as normal vector.
} 
Consequently, the first term $X$ of Eq. (11) is always null and the superbracket of the $\mathcal{A A}$ mechanism is reduced to:

$$
\left.S_{A A}=\left[b_{12} \underline{\mathrm{e}}_{1} \underline{\mathrm{e}}_{2} \mathrm{~b}_{34}\right]\left(\left[\mathrm{b}_{12} \underline{\mathrm{s}}_{1} \underline{\mathrm{s}}_{2} \dot{\mathrm{b}}_{5}\right] \underline{\underline{\mathrm{s}}}_{5} \underline{\mathrm{s}}_{3} \mathrm{~b}_{34} \underline{\mathrm{s}}_{4}\right]\right)
$$

The singularity conditions of the $\mathcal{A A}$ mechanism correspond to the vanishing conditions of term $Y$ that fall into two main cases:

(a) $\left[b_{12} \underline{e}_{1} \underline{e}_{2} b_{34}\right]=0 \Rightarrow$ points $b_{12}, b_{34}, \underline{e}_{1}$ and $\underline{e}_{2}$ are coplanar, which occurs if one of the following conditions is verified:

(a.1) $\mathrm{b}_{12} \equiv \mathrm{b}_{34}$, in this case points $B_{12}$ and $B_{34}$ coincide;

(a.2) $\mathrm{b}_{12} \equiv \underline{\mathrm{e}}_{1}$ (or $\left.\mathrm{b}_{12} \equiv \underline{\mathrm{e}}_{2}\right)$ : impossible as a finite line cannot coincide with a point at infinity;

(a.3) $\mathrm{b}_{34} \equiv \underline{\mathrm{e}}_{1}\left(\right.$ or $\left.\mathrm{b}_{34} \equiv \underline{\mathrm{e}}_{2}\right)$ : impossible;

(a.4) $\underline{\mathrm{e}}_{1} \equiv \underline{\mathrm{e}}_{2}$ : impossible because $\mathbf{e}_{1}$ and $\mathbf{e}_{2}$ are two fixed independent directions;

(a.5) Points $\mathrm{b}_{12}, \underline{\mathrm{e}}_{1}$ and $\underline{\mathrm{e}}_{2}$ are aligned: impossible as a finite point cannot be aligned with two distinct points at infinity;

(a.6) Points $\mathrm{b}_{34}, \underline{\mathrm{e}}_{1}$ and $\underline{\mathrm{e}}_{2}$ are aligned: impossible;

(a.7) Points $\mathrm{b}_{12}, \mathrm{~b}_{34}$ and $\underline{\mathrm{e}}_{1}$ are aligned: in this case $\left(B_{12} B_{34}\right)$ is parallel to $\mathbf{e}_{1}$ as shown in Fig. 7(a);

(a.8) Points $\mathrm{b}_{12}, \mathrm{~b}_{34}$ and $\underline{\mathrm{e}}_{2}$ are aligned: in this case $\left(B_{12} B_{34}\right)$ is parallel to $\mathbf{e}_{2}$;

(a.9) $\mathrm{b}_{12} \mathrm{~b}_{34} \wedge \underline{\mathrm{e}}_{1} \underline{\mathrm{e}}_{2}=0$ : in this case the point at infinity of line $\left(B_{12} B_{34}\right)$ belongs to the line at infinity $\underline{\mathrm{e}}_{1} \underline{\mathrm{e}}_{2}$. It means that line $\left(B_{12} B_{34}\right)$ lies in a plane having $\mathbf{e}_{1} \times \mathbf{e}_{2}$ as normal vector.

(b) $\left(b_{12} \underline{\mathbf{s}}_{1} \underline{\mathbf{s}}_{2}\right) \wedge\left(\underline{\mathbf{s}}_{3} b_{34} \underline{\mathbf{s}}_{4}\right) \wedge\left(b_{5} \underline{\mathbf{s}}_{5}\right)=0$, which occurs if one of the following conditions is verified:

(b.1) $\left(\mathrm{b}_{12} \underline{\mathbf{s}}_{1} \underline{\mathrm{s}}_{2}\right)=0 \Rightarrow$ plane $\left(\mathrm{b}_{12} \underline{\mathbf{s}}_{1} \underline{\mathbf{s}}_{2}\right)$ degenerates $\Rightarrow \underline{\mathbf{s}}_{1} \equiv \underline{\mathbf{s}}_{2}$, screws $\hat{\$}_{1}$ and $\hat{\$}_{2}$ are coaxial;

(b.2) $\left(\underline{\mathrm{s}}_{3} \mathrm{~b}_{34} \underline{\mathrm{s}}_{4}\right)=0 \Rightarrow$ screws $\hat{\$}_{3}$ and $\hat{\$}_{4}$ are coaxial;

(b.3) $\left(\mathrm{b}_{5} \underline{\mathrm{S}}_{5}\right)=0 \Rightarrow \mathrm{b}_{5} \equiv \underline{\mathbf{s}}_{2}$ : impossible;

(b.4) $\left(\mathrm{b}_{12} \underline{\mathrm{s}}_{1} \underline{\mathrm{s}}_{2}\right) \equiv\left(\underline{\mathrm{s}}_{3} \mathrm{~b}_{34} \underline{\mathrm{s}}_{4}\right) \Rightarrow \mathcal{V}_{12}$ and $\mathcal{V}_{34}$ are coplanar. Such a configuration is illustrated in Fig.77b);

(b.5) $\left(b_{5} \underline{\mathbf{S}}_{5}\right) \in\left(b_{12} \underline{\underline{s}}_{1} \underline{\underline{s}}_{2}\right) \Rightarrow$ the axis $A_{5}$ of screw $\hat{\$}_{5}$ lies in plane $\mathcal{V}_{12}$;

(b.6) $\left(b_{5} \underline{s}_{5}\right) \in\left(\underline{s}_{3} b_{34} \underline{s}_{4}\right) \Rightarrow A_{5}$ lies in $\mathcal{V}_{34}$;

(b.7) $\left(b_{5} \underline{s}_{5}\right)$, namely, the actuation force $\hat{\$}_{5}$ intersects the intersection line of planes $\left(b_{12} \underline{s}_{1} \underline{s}_{2}\right)$ and $\left(\underline{s}_{3} b_{34} \underline{s}_{4}\right)$, namely, $\left(\mathcal{V}_{12} \cap \mathcal{V}_{34}\right)$, as shown in Fig. $7(c)$. Thus, a singularity occurs if line $\left(b_{5} \underline{s}_{5}\right)$ of direction $\mathbf{s}_{5}$ and line $\left(\mathcal{V}_{12} \cap \mathcal{V}_{34}\right)$ of direction $\mathbf{e}_{2}$ intersect. A particular case of this condition, illustrated in Fig. 7(d), occurs if they intersect at infinity, i.e., if $\mathbf{s}_{5} \| \mathbf{e}_{2}$. This implies that $\rho_{5} \| \mathbf{e}_{2}, \rho_{5}$ being the direction of the actuated prismatic joint of the 5-th limb. Another particular case of this condition happens when line $\left(\mathcal{V}_{12} \cap \mathcal{V}_{34}\right)$ coincides with line $\left(b_{5} \underline{s}_{5}\right)$, i.e., with axis $A_{5}$.

From Eq. (15), a vector form for the singularity conditions can be expressed as follows:

$$
\left(\mathbf{n} \bullet\left(\mathbf{e}_{1} \times \mathbf{e}_{2}\right)\right)\left(\left(\left(\mathbf{s}_{1} \times \mathbf{s}_{2}\right) \times\left(\mathbf{s}_{3} \times \mathbf{s}_{4}\right)\right) \bullet \mathbf{s}_{5}\right)=0
$$

where $\mathbf{n}$ is the unit vector of line $\left(B_{12} B_{34}\right)$.

\subsection{Correspondence between GG and GCA}

This paper, in contrast with most of the literature dealing with the singularity analysis of PMs, does not reject one approach in favour of the other, i.e., GG and GCA, and it attempts to use both approaches as complementary rather than regarding them as opposites. To do so, we resort to the results obtained in [32] for the singularity analysis of the $\mathcal{A A}$ mechanism using the classification of linear varieties given in Table 2 that was presented in [9]. By virtue of the results obtained with GCA, the whole singularity conditions for the $\mathcal{A A}$ mechanism are made equivalent to the vanishing conditions of term $Y$. The latter can occur upon two different cases in which the first case falls into nine sub-cases, with five excluded cases, and the second case results in seven different cases where one was excluded. 


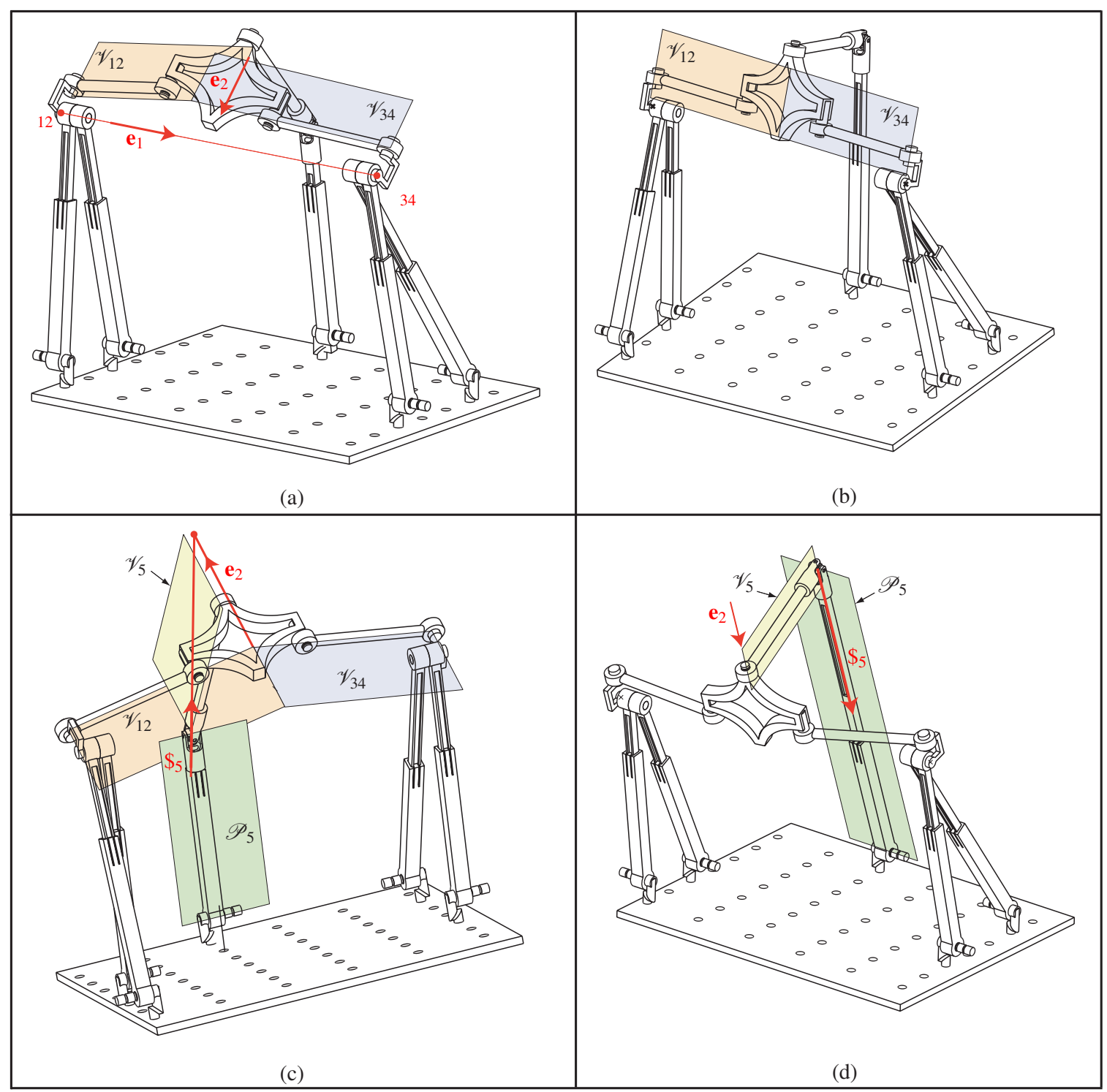

Figure 7: Singular configurations of the $\mathcal{A} A$ mechanism corresponding to cases (a.7), (b.4) and (b.7).

\subsubsection{The first case: (a.1), (a.7), (a.8) and (a.9)}

(a.1) If $B_{12} \equiv B_{34}$, then the four actuation forces $\hat{\$}_{1}, \hat{\$}_{2}, \hat{\$}_{3}$ and $\hat{\$}_{4}$ will intersect at a common point. In that case the foregoing forces span a variety of dimension three rather than four. From a theoretical stand point, such a configuration corresponds to condition 3c of GG. However, for the proposed design of the $\mathcal{A A}$ mechanism, this condition could be excluded.

(a.7) If $B_{12} B_{34} \| \mathbf{e}_{1}$, then the first axes (directed along $\mathbf{e}_{1}$ ) of the two $U$ joints of the simplified arrangements will coincide, as depicted in Fig. 7(a). By virtue of GG, in this case, two skew lines are transversal to five lines. Reaching this step, based on Fig. 7(a), it is straightforward to conclude that the intersection line of planes $\mathcal{V}_{12}$ and $\mathcal{V}_{34}$, namely, $\mathcal{T}_{v} \| \mathbf{e}_{2}$, is always concurrent with the axes of screws $\hat{\$}_{1}, \hat{\$}_{2}, \hat{\$}_{3}, \hat{\$}_{4}$ and $\hat{\$}_{c}$. On the other hand, when the first axes of both $U$ joints are pointed to each other, there is a second line, namely, $\left(B_{12} B_{34}\right) \| \mathbf{e}_{1}$, which crosses the foregoing five screws and which is skew with the first transversal line, $\mathcal{T}_{v}$, described above. Accordingly, this singularity is referred to as hyperbolic congruence, condition $4 \mathrm{~b}$ of $\mathrm{GG}$, for which two skew lines are transversal to all lines. In such a configuration, the five screws $\hat{\$}_{1}, \hat{\$}_{2}, \hat{\$}_{3}, \hat{\$}_{4}$ and $\hat{\$}_{c}$ span a variety of dimension four rather than five.

(a.8) To explain this singularity, namely, $B_{12} B_{34} \| \mathbf{e}_{2}$, one should think beyond the classification given in [9]. From the classification proposed in [19] for some special cases in which a line at infinity is among a set of Plücker lines, it reveals that this singularity corresponds to condition $\mathcal{C}_{4}^{2 \infty}$. In this condition, two pairs 
of lines, namely, $\left\{A_{1}, A_{2}\right\}$ (the axes of $\hat{\$}_{1}$ and $\hat{\$}_{2}$ ) and $\left\{A_{3}, A_{4}\right\}$ (the axes of $\hat{\$}_{3}$ and $\hat{\$}_{4}$ ), span two distinct planes $\mathcal{V}_{12}$ and $\mathcal{V}_{34}$ whose projections into the plane perpendicular to the line at infinity, $\hat{\$}_{c}$, are parallel. For the proposed design of the $\mathcal{A} \mathcal{A}$ mechanism, this condition cannot occur.

(a.9) This case is a general condition that covers (a.7) and (a.8). From the above analysis for (a.7) and (a.8), it can be inferred that all the configurations that condition (a.9) holds can be classified as hyperbolic congruence, unless condition (a.8) is true. However, it can be noticed that $\mathcal{C}_{4}^{2 \infty}$ is the general condition of (a.9).

Table 2: Singularity conditions of the $\mathcal{A A}$ mechanism: correspondence between GCA and GG.

\begin{tabular}{|c|c|c|}
\hline $\begin{array}{l}\text { Singularity } \\
\text { case (GCA) }\end{array}$ & $\begin{array}{c}\text { Geometric } \\
\text { singularity condition }\end{array}$ & $\begin{array}{l}\text { Corresponding GLG } \\
\text { singularity type }\end{array}$ \\
\hline (a.1) & $B_{12} \equiv B_{34}$ & 3c, all lines through a point \\
\hline (a.7) & $\left(B_{12} B_{34}\right) \| \mathbf{e}_{1}$ & 4b, hyperbolic congruence \\
\hline (a.8) & $\left(B_{12} B_{34}\right) \| \mathbf{e}_{2}$ & \multirow{2}{*}{$\begin{array}{l}\mathcal{C}_{4}^{2 \infty}, \text { two pairs of concurrent lines } \\
\text { plus a line at infinity }\end{array}$} \\
\hline (a.9) & $\left(B_{12} B_{34}\right) \perp \mathbf{e}_{1} \times \mathbf{e}_{2}$ & \\
\hline (b.1) & \multirow{2}{*}{$\begin{array}{l}A_{1} \equiv A_{2} \\
A_{3} \equiv A_{4}\end{array}$} & \multirow[t]{2}{*}{1, point } \\
\hline (b.2) & & \\
\hline (b.4) & $\mathcal{V}_{12} \equiv \mathcal{V}_{34}$ & $\begin{array}{l}3 \mathrm{~d}, \text { all lines in a plane } \\
5 \mathrm{~b}, \text { singular linear complex }\end{array}$ \\
\hline (b.5) & $A_{5} \in \mathcal{V}_{12}$ & \multirow{4}{*}{$5 \mathrm{~b}$, singular linear complex } \\
\hline (b.6) & $A_{5} \in \mathcal{V}_{34}$ & \\
\hline \multirow{3}{*}{ (b.7) } & $\left(\mathcal{V}_{12} \cap \mathcal{V}_{34}\right) \equiv A_{5}$ & \\
\hline & $\mathbf{s}_{5} \| \mathbf{e}_{2}$ & \\
\hline & $\left(\mathcal{V}_{12} \cap \mathcal{V}_{34}\right) \cap A_{5} \neq \varnothing$ & $\begin{array}{l}5 \mathrm{~b}, \text { singular linear complex } \\
4 \mathrm{c}, \text { parabolic congruence }\end{array}$ \\
\hline
\end{tabular}

\subsubsection{The second case: (b.1), (b.2), (b.4), (b.5), (b.6) and (b.7)}

(b.1) Only two conditions lead to coaxial $\hat{\$}_{1}$ and $\hat{\$}_{2}$ : (a) $\mathcal{P}_{1} \equiv \mathcal{P}_{2}$ and (b) $\mathbf{v}_{12} \| \mathbf{e}_{1}$ (this is also a limb singularity 5 where the actuation wrench becomes reciprocal to the input). In such a configuration, $A_{1}=$ $\left(\mathcal{P}_{1} \cap \mathcal{V}_{12}\right)$ coincides with $A_{2}=\left(\mathcal{P}_{2} \cap \mathcal{V}_{12}\right)$, namely, the three planes $\mathcal{P}_{1}, \mathcal{P}_{2}$ and $\mathcal{V}_{12}$ intersect at a common line.

(b.2) Based on the above discussion, coaxial $\hat{\$}_{3}$ and $\hat{\$}_{4}$ happens upon two configurations: (a) $\mathcal{P}_{3} \equiv \mathcal{P}_{4}$ and (b) $\mathbf{v}_{34} \| \mathbf{e}_{1}$ (this is also a limb singularity).

(b.4) When $\mathcal{V}_{12} \equiv \mathcal{V}_{34}$, axes $A_{1}, A_{2}, A_{3}$ and $A_{4}$ lie in a common plane and the screw system given by $\operatorname{span}\left(\hat{\$}_{1}, \hat{\$}_{2}, \hat{\$}_{3}, \hat{\$}_{4}\right)$ degenerates. Such a configuration is illustrated in Fig. 7 (b) and corresponds to condition $3 \mathrm{~d}$ of GG, all lines in a plane. This case also corresponds to condition $5 \mathrm{~b}$ of GG since in that case the intersection line of $\mathcal{V}_{12}$ and $\mathcal{V}_{5}$, which is of direction $\mathbf{e}_{2}$, intersects the six screws of the overall wrench system.

(b.5) We know that line $\left(\mathcal{V}_{12} \cap \mathcal{V}_{34}\right)$ is always concurrent with the axes of screws $\hat{\$}_{1}, \hat{\$}_{2}, \hat{\$}_{3}$ and $\hat{\$}_{4}$. As mentioned previously, this line, being of direction $\mathbf{e}_{2}$, passes through the point at infinity $\underline{e}_{1}$. Therefore, it crosses the line at infinity $\hat{\$}_{c}=\underline{e}_{1} \underline{e}_{2}$. If $\left(b_{5} \underline{s}_{5}\right) \in\left(b_{12} \underline{s}_{1} \underline{s}_{2}\right)$, then line $\left(\mathcal{V}_{12} \cap \mathcal{V}_{34}\right)$ also crosses screw $\hat{\$}_{5}$ and, as a result, in such a configuration, this line crosses the six screws $\hat{\$}_{1}, \ldots, \hat{\$}_{5}$, and $\hat{\$}_{c}$. Based on GG, this singularity is referred to as singular linear complex, condition $5 \mathrm{~b}$, which corresponds to a variety of dimension five.

\footnotetext{
${ }^{5}$ Based on the results presented in [32], such a mechanism exhibits a limb singularity when $\mathbf{v}_{12} \| \mathbf{e}_{1}$ or $\rho_{i}=0$.
} 
(b.6) This case is similar to (b.5).

(b.7) In this case, $\hat{\$}_{5}$ passes through the intersection of two pairs of planes spanned by the set $\left\{A_{1}, A_{2}, A_{3}, A_{4}\right\}$ of the axes of screws $\hat{\$}_{1}, \hat{\$}_{2}, \hat{\$}_{3}$ and $\hat{\$}_{4}$. There are several possibilities for which this set can span different pairs of planes. All these possibilities are studied in [32] and three cases turn out to be possible:

1. All planes $\mathcal{P}_{i}, i=1, \ldots, 5$, have a common intersection line. Clearly, this line called $\mathcal{T}_{p}$ is parallel to $\mathbf{e}_{1}$. Thus, it is transversal to the line associated with the constraint wrench $\hat{\$}_{c}$. This singularity is referred to as singular linear complex, condition $5 \mathrm{~b}$.

2. All planes $\mathcal{V}_{i}, i=1, \ldots, 5$, have a common intersection line. Obviously, this line called $\mathcal{T}_{v}$ is parallel to $\mathbf{e}_{2}$ and transversal to $\hat{\$}_{c}$. This singularity is referred to as singular linear complex, condition 5b. As mentioned previously, there is a particular configuration: $\mathbf{s}_{5} \| \mathbf{e}_{2}$, which implies that $\rho_{5} \| \mathbf{e}_{2}$. Such a configuration is illustrated in Fig. 7(d).

3. $\hat{\$}_{5}$ intersects the line passing through $\left(B_{12} B_{34}\right)$. This is a parabolic congruence, condition $4 \mathrm{c}$, where the lines associated with $\hat{\$}_{1}, \hat{\$}_{2}, \hat{\$}_{3}$ and $\hat{\$}_{4}$ belong to the union of three planar pencils of lines, in different planes, having a common line.

Table 2 illustrates the valid geometric singularity conditions of the $\mathcal{A} \mathcal{A}$ mechanism obtained with GCA as well as their corresponding GG singularity type.

\subsection{Instantaneous motions associated with parallel singularities}

Parallel singularities are critical configurations in which a PM loses its inherent stiffness and the motion of its end-effector becomes uncontrollable. Since the $\mathcal{A} \mathcal{A}$ mechanism is free of constraint singularities, its actuation singularities correspond to the rank deficiency of $\mathbf{J}$. In such configurations, the actuators of the PM cannot control its end-effector's motion, namely, the end-effector might generate some infinitesimal motion(s) even when the actuators are locked. In each of these singularities, the wrenches $\hat{\$}_{1}, \hat{\$}_{2}, \hat{\$}_{3}, \hat{\$}_{4}, \hat{\$}_{5}$ and $\hat{\$}_{c}$ form a $n<6$-system and the twist(s) reciprocal to this system for a given actuation singularity determine(s) the uncontrolled motion of the end-effector [16].

(a.1) Theoretically, this corresponds to a configuration in which the four actuation forces $\$_{i}(i=1, \ldots 4)$ pass through a common point, namely, $B_{12} \equiv B_{34}$. In that case, there exists a finite line $\left(B_{12} B_{5}\right)$, which crosses the five actuation forces $\$_{i}(i=1, \ldots 4)$. Let $\mathbf{u}$ and $\underline{u}=(\mathbf{u} ; 0)$ be the unit vector and the point at infinity of line $B_{12} B_{5}$, respectively. Two cases turn to be possible:

- $\underline{\underline{u}} \in \underline{\mathrm{e}}_{1} \underline{\mathrm{e}}_{2} \Leftrightarrow$ line $\left(B_{12} B_{5}\right)$ is orthogonal to $\mathbf{e}_{1} \times \mathbf{e}_{2}$, i.e., it crosses $\hat{\$}_{c}$ in addition to the five actuation forces. In that case, the uncontrolled motion corresponds to a zero-pitch twist given by $\left(\mathbf{u} ; \mathbf{r}_{B_{12}} \times \mathbf{u}\right)$. Thus, it is a pure rotation about an axis of direction $\mathbf{u}$;

- $\underline{u} \notin \underline{\mathrm{e}}_{1} \underline{\mathrm{e}}_{2}$. In that case, one can find neither a zero-pitch twist nor an infinite-pitch twist reciprocal to screws $\$_{1}, \ldots, \$_{5}$ and $\$_{c}$. Thus, the uncontrolled motion corresponds to a finite-pitch twist, namely, a combination of a rotation and a translation.

(a.7),(a.8) and (a.9) A general condition for these cases is $\left(B_{12} B_{34}\right) \perp \mathbf{e}_{1} \times \mathbf{e}_{2}$, which implies that a finite line $\left(B_{12} B_{34}\right)$ crosses the five screws $\hat{\$}_{1}, \hat{\$}_{2}, \hat{\$}_{3}, \hat{\$}_{4}$ and $\hat{\$}_{c}$ that form a 4 -system. However, in these cases, line $\left(B_{12} B_{34}\right)$ does not generally intersect $\hat{\$}_{5}$. It can be concluded that the uncontrolled motion corresponds to a finite-pitch twist.

(b.1) In this condition, screws $\hat{\$}_{1}$ and $\hat{\$}_{2}$ are coaxial. It could be noticed that the condition $\mathcal{P}_{1} \equiv \mathcal{P}_{2}$ appears to be impossible for the proposed mechanism. On the other hand, if $\mathbf{v}_{12} \| \mathbf{e}_{1}$, the mechanisms exhibits simultaneously an actuation singularity $\left(\hat{\$}_{1}\right.$ and $\hat{\$}_{2}$ are coaxial) and two limb singularities $\left(\hat{\$}_{1}\right.$ and $\hat{\$}_{2}$ are reciprocal to the twists associated with the actuated prismatic joints of the first and the second limbs). As $\mathbf{s}_{1}\left\|\mathbf{s}_{2}\right\| \mathbf{e}_{1}$ in such a configuration, the mechanism loses the translational DOF along $\mathbf{e}_{1}$.

(b.2) This condition can be analyzed similarly to (b.1). 
(b.4) In that case a line in plane $\mathcal{V}_{12} \equiv \mathcal{V}_{34}$ of direction $\mathbf{e}_{2}$ is reciprocal to the five screws $\hat{\$}_{1}, \hat{\$}_{2}, \hat{\$}_{3}, \hat{\$}_{4}$ and $\hat{\$}_{c}$ but not necessarily reciprocal to $\hat{\$}_{5}$. Thus, this case is similar to (a.9), namely, the uncontrolled motion corresponds to a finite-pitch twist.

(b.5),(b.6) and (b.7) In these cases, line $\left(\mathcal{V}_{12} \cap \mathcal{V}_{34}\right)$ crosses axis $A_{5}$ of screw $\hat{\$}_{5}$. As mentioned previously, this implies that line $\left(\mathcal{V}_{12} \cap \mathcal{V}_{34}\right)$ of direction $\mathbf{e}_{2}$ crosses the six wrenches $\hat{\$}_{1}, \hat{\$}_{2}, \hat{\$}_{3}, \hat{\$}_{4}, \hat{\$}_{5}$ and $\hat{\$}_{c}$. Accordingly, in all of these cases, the actuators cannot control the rotational DOF of the moving platform about an axis of direction $\mathbf{e}_{2}$.

\section{Singularity Analysis of the $\mathcal{B B}$ mechanism}

\subsection{Superbracket of the $\mathcal{B B}$ mechanism}

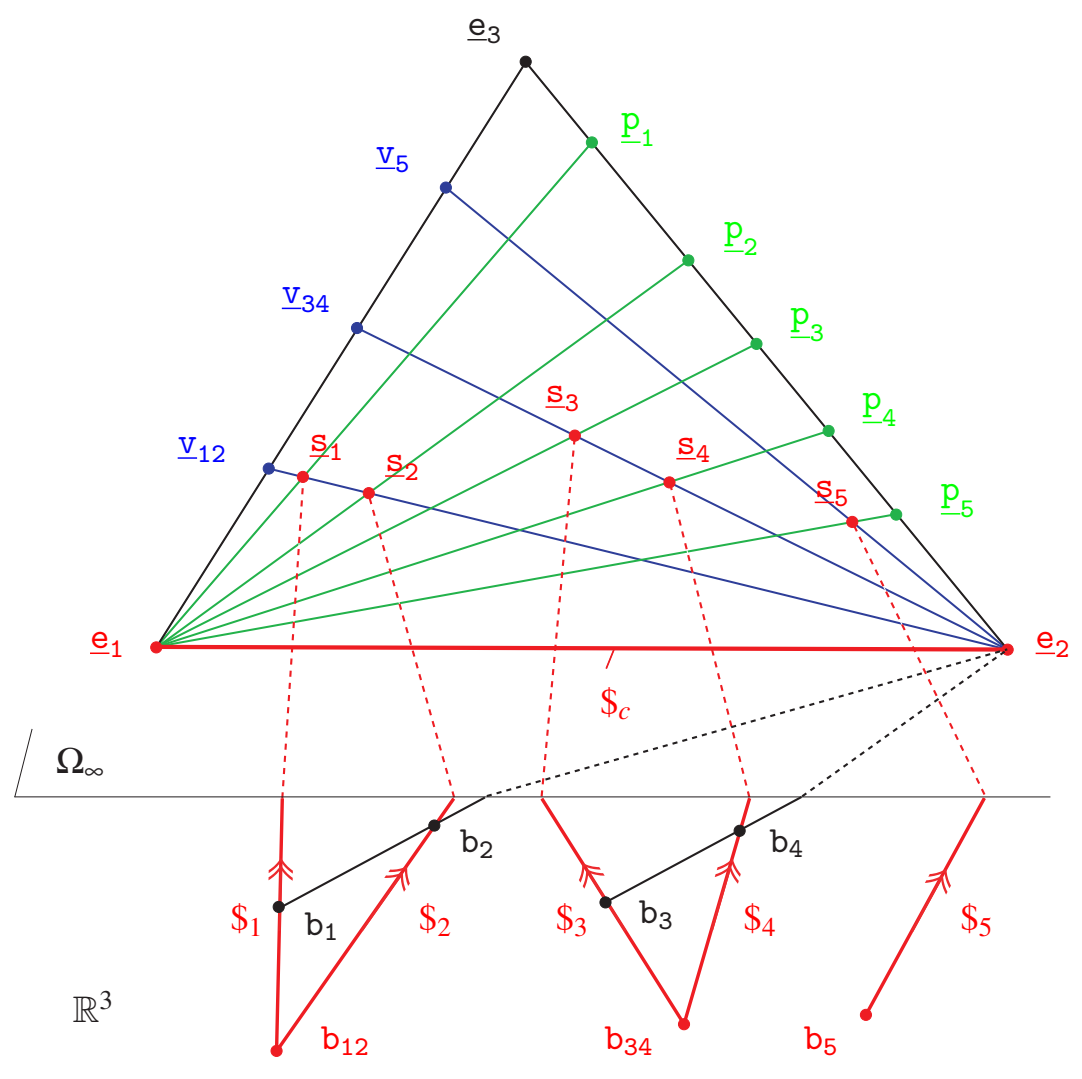

Figure 8: Wrench graph of the $\mathcal{B B}$ mechanism in $\mathbb{P}_{3}$.

For the $\mathcal{B B}$ mechanism, extensors $\underline{\mathbf{s}}_{1}, i=1, \ldots, 5$, are obtained similarly to the $\mathcal{A A}$ mechanism. Let $\mathrm{b}_{12}, \mathrm{~b}_{34}, \mathrm{~b}_{1}, \mathrm{~b}_{2}, \mathrm{~b}_{3}, \mathrm{~b}_{4}$ and $\mathrm{b}_{5}$ be the 1-extensors associated with points $B_{12}, B_{34}, B_{1}, B_{2}, B_{3}, B_{4}$ and $B_{5}$, respectively. Since lines $\left(B_{1} B_{2}\right)$ and $\left(B_{3} B_{4}\right)$ are parallel to $\mathbf{e}_{2}, \mathrm{~b}_{1} \mathrm{~b}_{2}$ and $\mathrm{b}_{3} \mathrm{~b}_{4}$ intersect $\Omega_{\infty}$ at $\underline{\mathrm{e}}_{2}$. Figure 8 shows the wrench graph of the $\mathcal{B B}$ mechanism in $\mathbb{P}_{3}$.

The projective line associated with $\hat{\$}_{c}=\left(\mathbf{0} ; \mathbf{e}_{1} \times \mathbf{e}_{2}\right)$ passes through the points at infinity $\underline{\mathrm{e}}_{1}$ and $\underline{\mathrm{e}}_{2}$. Similarly to the $\mathcal{A A}$ mechanism, screw $\hat{\$}_{5}$ is represented by $\mathrm{b}_{5} \underline{\mathbf{s}}_{5}$, where $\underline{\mathbf{s}}_{5}=\left(\mathbf{s}_{5} ; 0\right)$ is the point at infinity of direction $\mathbf{s}_{5}$. In what concerns screws $\hat{\$}_{i}(i=1, \ldots, 4)$, several representations turn to be possible. As an example, let us consider screw $\hat{\$}_{1}$. Since the projective line associated with this screw passes through points $b_{1}$, $\mathrm{b}_{12}$ and $\underline{\mathrm{s}}_{1}$, the 2-extensor associated with this screw can be expressed as the join of two among these three points. The superbracket must be chosen in such a way that we obtain the simplest expression. Therefore, all relations between the corresponding projective elements must be considered. Since $b_{12}$ is a common point of $\hat{\$}_{1}$ and $\hat{\$}_{2}$, it must be part of the two points representing $\hat{\$}_{1}$ in the superbracket. Thus, the second point is either $b_{1}$ or $\underline{s}_{1}$. Since $b_{1}$ and $b_{2}$ are aligned with $\underline{e}_{2}$ as well as $b_{3}$ and $b_{4}$, we choose to include points $b_{i}$ in the superbracket of this PM rather than $\underline{\underline{s}}_{i}, i=1, \ldots, 4$. However, for the superbracket of the $\mathcal{B B}$ mechanism, the use of $\mathrm{b}_{\mathrm{i}}$ or $\underline{\mathrm{s}}_{\mathrm{i}}, i=1, \ldots, 4$, leads to the same number of monomials in the simplified expression. Accordingly, 
the superbracket of the $\mathcal{B B}$ mechanism takes the form:

$$
\begin{aligned}
S_{B B} & =\left[b_{12} b_{1}, b_{12} b_{2}, b_{5} \underline{s}_{5}, \underline{e}_{1} \underline{e}_{2}, b_{34} b_{3}, b_{34} b_{4}\right]=\left[b_{12} \underline{s}_{1}, b_{12} \underline{s}_{2}, b_{5} \underline{s}_{5}, \underline{e}_{1} \underline{e}_{2}, b_{34} \underline{\mathbf{s}}_{3}, b_{34} \underline{\mathbf{s}}_{4}\right] \\
& =\left[b_{12} \underline{e}_{1} \underline{e}_{2} b_{34}\right]\left(\left[b_{12} b_{1} b_{2} \dot{b}_{5}\right]\left[\underline{\dot{s}}_{5} b_{3} b_{34} b_{4}\right]\right)-\left[b_{12} b_{5} \underline{s}_{5} b_{34}\right]\left(\left[b_{12} b_{1} b_{2} \underline{\dot{e}}_{1}\right]\left[\underline{e}_{2} b_{3} b_{34} b_{4}\right]\right) \\
& =\left[b_{12} \underline{e}_{1} \underline{e}_{2} b_{34}\right]\left(\left[b_{12} b_{1} b_{2} \dot{b}_{5}\right]\left[\underline{\dot{s}}_{5} b_{3} b_{34} b_{4}\right]\right)
\end{aligned}
$$

The singularity conditions of the $\mathcal{B B}$ mechanism correspond to the vanishing conditions of $S_{B B}$ that fall into two main cases:

(a) $\left[\mathrm{b}_{12} \underline{\mathrm{e}}_{1} \underline{\mathrm{e}}_{2} \mathrm{~b}_{34}\right]=0 \Rightarrow$ points $\mathrm{b}_{12}, \mathrm{~b}_{34}, \underline{\mathrm{e}}_{1}$ and $\underline{\mathrm{e}}_{2}$ are coplanar, which can occur upon one of the following subcases:

(a.1) Points $B_{12}$ and $B_{34}$ coincide;

(a.2) Line $\left(B_{12} B_{34}\right)$ is parallel to $\mathbf{e}_{1}$;

(a.3) Line $\left(B_{12} B_{34}\right)$ is parallel to $\mathbf{e}_{2}$;

(a.4) Line $\left(B_{12} B_{34}\right)$ is orthogonal to $\mathbf{e}_{1} \times \mathbf{e}_{2}$.

(b) $\left(b_{12} b_{1} b_{2}\right) \wedge\left(b_{3} b_{34} b_{4}\right) \wedge\left(b_{5} \underline{s}_{5}\right)=0$, which occurs if one of the following conditions is verified:

(b.1) Points $B_{12}, B_{1}$ and $B_{2}$ are aligned (or two of them coincide), in this case, screws $\hat{\$}_{1}$ and $\hat{\$}_{2}$ are coaxial;

(b.2) Screws $\hat{\$}_{3}$ and $\hat{\$}_{4}$ are coaxial;

(b.3) Planes $\mathcal{V}_{12}$ and $\mathcal{V}_{34}$ coincide;

(b.4) Axis $A_{5}$ of screw $\hat{\$}_{5}$ lies in plane $\mathcal{V}_{12}$;

(b.5) $A_{5}$ lies in $\mathcal{V}_{34}$

(b.6) The intersection line of planes $\mathcal{V}_{12}$ and $\mathcal{V}_{34}$ coincides with $A_{5}$;

(b.7) $A_{5}$ is directed along $\mathbf{e}_{2}$, i.e., $\mathbf{s}_{5}$ is parallel to $\mathbf{e}_{2}$. This implies that $\rho_{5} \| \mathbf{e}_{2}$;

(b.8) $\left(\mathcal{V}_{12} \cap \mathcal{V}_{34}\right)$ and $A_{5}$ are concurrent.

As mentioned in Sec. 3.2.2, for the $\mathcal{B B}$ mechanism, point $B_{12}$ (respectively $B_{34}$ ) belongs to the axis, directed along $\mathbf{e}_{1}$, of the $\mathrm{R}$-joint centered at $A_{12}$ (respectively $A_{34}$ ) and attached to the fixed base. Thus, the second and third Cartesian coordinates of point $B_{12}$ (respectively $B_{34}$ ) are the same as those of point $A_{12}$ (respectively $A_{34}$ ). As pointed out in [33], a $\mathcal{B B}$ mechanism has a singular orientation for $\theta$, called $\theta_{s}$, which depends on the geometric parameters of the base and can be found by expanding condition (a.4):

$$
\left(y_{A_{12}}-y_{A_{34}}\right) \sin \theta_{s}+\left(z_{A_{12}}-z_{A_{34}}\right) \cos \theta_{s}=0
$$

where $\left[x_{A_{12}}, y_{A_{12}}, z_{A_{12}}\right]^{T}$ and $\left[x_{A_{34}}, y_{A_{34}}, z_{A_{34}}\right]^{T}$ are the Cartesian coordinate vectors of points $A_{12}$ and $A_{34}$, respectively. For instance, for the design proposed in Fig. 4: $\theta_{s}=0$.

\section{Conclusions}

This paper presented an exhaustive singularity analysis of two 3T2R 5-DOF Parallel Mechanisms (PMs) having a simplified architecture. The proposed singularity analysis approach is applicable to lower-mobility PMs, mainly those having infinite-pitch wrench(es) among the rows of their Jacobian matrix. For a given PM, this approach consists of three main steps:

(i) The use of screw theory to represent the wrenches of the PM in the projective space. This representation is very useful since it illustrates all the geometric relations between the wrenches of the PM and allows the simplification of the superbracket decomposition. 
(ii) The use of Grassmann-Cayley Algebra (GCA) to enumerate all the singularity conditions of the PMs under study and to derive a vector expression for the singularity locus, which is difficult to obtain for such PMs with other singularity analysis methods.

(iii) The use of Grassmann Geometry (GG) to examine the rank deficiency of the Jacobian matrix and characterize the uncontrolled motion for a given singularity condition.

This paper showed that GCA and GG could be used together in order to better characterize and investigate the singularities of spatial parallel mechanisms. The proposed approach is currently used by the authors to analyze the singularities of other types of 5-DOF PMs like 3R2T PMs.

\section{Acknowledgments}

The authors would like to acknowledge the financial support of the Natural Sciences and Engineering Research Council of Canada (NSERC), the Canada Research Chair program and the French "Agence Nationale de la Recherche" (Project "SiRoPa", SIngularités des RObots PArallèles).

\section{References}

[1] J. P. Merlet. Singular Configurations of Parallel Manipulators and Grassmann Geometry. The International Journal of Robotics Research, 8(5):45-56, 1989.

[2] P. Ben-Horin and M. Shoham. Singularity Analysis of a Class of Parallel Robots Based on GrassmannCayley Algebra. Mechanism and Machine Theory, 41(8):958-970, 2006.

[3] C. Gosselin and J. Angeles. Singularity Analysis of Closed-Loop Kinematic Chains. IEEE Transactions on Robotics and Automation, 6(3):281-290, 1990.

[4] Y. Fang and L. W. Tsai. Structure Synthesis of a Class of 4-DoF and 5-DoF Parallel Manipulators with Identical Limb Structures. The International Journal of Robotics Research, 21(9):799-810, 2002.

[5] M. Conconi and M. Carricato. A New Assessment of Singularities of Parallel Kinematic Chains. In Advances in Robot Kinematics: Analysis and Design, pages 3-12, 2008.

[6] D. Zlatanov, R. G. Fenton, and B. Benhabib. Singularity Analysis of Mechanisms and Robots Via a Velocity-Equation Model of the Instantaneous Kinematics. In IEEE International Conference on Robotics and Automation, pages 986-991, San Diego, CA, 1994.

[7] D. Zlatanov, I. Bonev, and C. M. Gosselin. Constraint Singularities of Parallel Mechanisms. In IEEE International Conference on Robotics and Automation, pages 496-502, Washington, D.C., May 11 Û15 2002.

[8] S. A. Joshi and L. W. Tsai. Jacobian Analysis of Limited-DOF Parallel Manipulators. ASME Journal of Mechanical Design, 124(2):254-258, June 2002.

[9] J. P. Merlet. Parallel Robots, volume 128 of Solid Mechanics and Its Applications. Springer, 2006.

[10] T. Mbarek, G. Lonij, and B. Corves. Singularity Analysis of a Fully Parallel Manipulator with FiveDegrees-of-Freedom Based on Grassmann Line Geometry. In 12th IFToMM World Congress, BesanAgon, France, June 18-21 2007.

[11] P. Ben-Horin and M. Shoham. Application of Grassmann-Cayley Algebra to Geometrical Interpretation of Parallel Robot Singularities. The International Journal of Robotics Research, 28(1):127-141, 2009.

[12] N. L. White. Grassmann-Cayley Algebra and Robotics. Journal of Intelligent and Robotic Systems, 11(1):91-107, 1994. 
[13] B. Monsarrat and C. Gosselin. Singularity Analysis of a Three-leg Six-Degree-Of-Freedom Parallel Platform Mechanism Based on Grassmann Line Geometry. The International Journal of Robotics Research, 20(4):312-328, 2001.

[14] E. Staffetti and F. Thomas. Kinestatic Analysis of Serial and Parallel Robot Manipulators Using Grassmann-Cayley Algebra. In J. Lenarčič and M. M. Stanišić, editors, Advances in Robot Kinematics, pages 17-26, 2000.

[15] T. K. Tanev. Geometric Algebra Approach to Singularity of Parallel Manipulators with Limited Mobility. In Advances in Robot Kinematics, pages 8-39. Springer, 2008.

[16] A. Wolf and M. Shoham. Investigation of Parallel Manipulators using Linear Complex Approximation. ASME Journal of Mechanical Design, 125:564-572, 2003.

[17] M. Tale Masouleh, C. Gosselin, M. Husty, and D. R. Walter. Forward Kinematic Problem of 5-RPUR Parallel Mechanisms (3T2R) with Identical Limb Structures. Mechanism and Machine Theory, 46(7):945 -959wolf2006screw, 2011.

[18] M. Tale Masouleh, M. Husty, and C. Gosselin. Forward Kinematic Problem of 5-PRUR Parallel Mechanisms Using Study Parameters. In Advances in Robot Kinematics: Motion in Man and Machine, pages 211-221. Springer, 2010.

[19] M. Tale Masouleh. Kinematic Analysis of Five-DOF (3T2R) Parallel Mechanisms with Identical Limb Structures. PhD thesis, Laval University, Quebec, QC, Canada, September 2010.

[20] X. Kong and C. Gosselin. Type Synthesis of Parallel Mechanisms, volume 33. Springer, Heidelberg, 2007.

[21] F. Gao, B. Peng, H. Zhao, and W. Li. A Novel 5-DOF Fully Parallel Kinematic Machine Tool. The International Journal of Advanced Manufacturing Technology, 31(1):201-207, 2006.

[22] O. Piccin, B. Bayle, B. Maurin, and M. de Mathelin. Kinematic Modeling of a 5-DOF Parallel Mechanism for Semi-Spherical Workspace. Mechanism and Machine Theory, 44(8):1485-1496, 2009.

[23] Z. Huang and Q. C. Li. General Methodology for Type Synthesis of Symmetrical Lower-Mobility Parallel Manipulators and Several Novel Manipulators. The International Journal of Robotics Research, 21(2):131-145, 2002.

[24] Z. Huang and Q. C. Li. Type Synthesis of Symmetrical Lower-Mobility Parallel Mechanisms using the Constraint-Synthesis Method. The International Journal of Robotics Research, 22(1):59-79, 2003.

[25] X. Kong and C. Gosselin. Type Synthesis of 5-DOF Parallel Manipulators Based on Screw Theory. Journal of Robotic Systems, 22(10):535-547, 2005.

[26] S. J. Zhu and Z. Huang. Eighteen Fully Symmetrical 5-DoF 3R2T Parallel Manipulators with Better Actuating Modes. The International Journal of Advanced Manufacturing Technology, 34(3):406-412, 2007.

[27] Z. Huang and Q. C. Li. Two Novel Symmetrical 5-DOF Parallel Mechanisms. Journal of Yanshan University, 25(4):283-286, 2001.

[28] Q. Jin, T. L. Yang, A. X. Liu, H. P. Shen, and F. H. Yao. Structure Synthesis of a Class of 5-DOF Parallel Robot Mechanisms Based on Single Opened-Chain Units. In ASME 2001 Design Engineering Technical Conferences, number DAC21153 in DETC2001, Pittsburgh, PA, 2001.

[29] T. Mbarek, I. Barmann, and B. Corves. Fully Parallel Structures with Five Degree of Freedom: Systematic Classification and Determination of Workspace. In Proceedings of Mechatronics \& Robotics, pages 990996, Aachen, 2004.

[30] J. Wang and C. Gosselin. Kinematic Analysis and Singularity Representation of Spatial Five-Degree-ofFreedom Parallel Mechanisms. Journal of Robotic Systems, 14(12):851-869, 1997. 
[31] C. Gosselin, M. Tale Masouleh, V. Duchaine, P. L. Richard, S. Foucault, and X. Kong. Parallel Mechanisms of the Multipteron Family: Kinematic Architectures and Benchmarking. In IEEE International Conference on Robotics and Automation, pages 555-560, Roma, Italy, April 2007.

[32] M. Tale Masouleh and C. Gosselin. Singularity Analysis of 5-RPRRR Parallel Mechanisms via Grassmann Line Geometry. In Proceedings of the 2009 ASME Design Engineering Technical Conferences, number 86261 in DETC2009, 2009.

[33] M. Tale Masouleh, M. H. Saadatzi, C. Gosselin, and H. D. Taghirad. A Geometric Constructive Approach for the Workspace Analysis of Symmetrical 5-PRUR Parallel Mechanisms (3T2R). In ASME Design Engineering Technical Conferences, number 28509 in DETC2010, Montreal, Quebec, Canada, August 15-18 2010.

[34] M. Tale Masouleh and C. Gosselin. Kinematic Analysis and Singularity Representation of 5-RPRRR Parallel Mechanisms. In Fundamental Issues and Future Research Directions for Parallel Mechanisms and Manipulators, pages 79-90, Montpellier, France, September 21-22 2008.

[35] D. Kanaan, P. Wenger, S. Caro, and D. Chablat. Singularity Analysis of Lower-Mobility Parallel Manipulators using Grassmann-Cayley Algebra. IEEE Transactions on Robotics, 25:995-1004, 2009.

[36] S. Amine, D. Kanaan, S. Caro, and P. Wenger. Singularity Analysis of Lower-Mobility Parallel Robots with an Articulated Nacelle. In Advances in Robot Kinematics: Motion in Man and Machine 2010, Part 5, pages 273-282. Springer, 2010.

[37] S. Amine, D. Kanaan, S. Caro, and P. Wenger. Constraint and Singularity Analysis of Lower-Mobility Parallel Manipulators with Parallelogram Joints. In ASME 2010 International Design Engineering Technical Conferences, number 28483 in DETC2010, Montreal, Quebec, Canada, August 15-18 2010.

[38] R. S. Ball. A Treatise On the Theory of Screws. Cambridge University Press, Cambridge, CA, 1900.

[39] K. J. Waldron. The Mobility of Linkages. PhD Thesis, Stanford University, Cambridge, CA, 1969.

[40] K. H. Hunt. Kinematic Geometry of Mechanisms. Clarendon Press, Oxford, 1978.

[41] A. Wolf and M. Shoham. Screw theory tools for the synthesis of the geometry of a parallel robot for a given instantaneous task. Mechanism and Machine Theory, 41(6):945 -959, 2006.

[42] N. L. White. Grassmann-Cayley Algebra and Robotics Applications, volume VIII. Handbook of Geometric Computing, 2005.

[43] T. Huang, H. T. Liu, and D. G. Chetwynd. Generalized Jacobian analysis of lower mobility manipulators. Mechanism and Machine Theory, 46(6):831-844, 2011.

[44] T. McMillan. Invariants of Antisymmetric Tensors. PhD Thesis, University of Florida, 1990.

[45] J. P. Merlet. Parallel Manipulators. Part 2: Theory. Singular Configurations and Grassmann Geometry. Technical Report 791, INRIA, Sophia Antipolis, France, 1988.

[46] I. A. Bonev and C. M. Gosselin. Geometric Algorithms for the Computation of the Constant-Orientation Workspace and Singularity Surface of a Special 6-RUS Parallel Manipulator. In ASME 2002 Design Engineering Technical Conferences, number MECH-34257 in DETC2002, Montreal, Quebec, Canada, September 29-October 22002. 